\title{
Measuring slepton masses and mixings at the LHC
}

\author{
Jonathan L. Feng, ${ }^{a}$ Sky T. French, ${ }^{b}$ Iftah Galon, ${ }^{c}$ Christopher G. Lester, ${ }^{b}$ Yosef Nir, ${ }^{d}$ \\ Yael Shadmi, ${ }^{a, c}$ David Sanford ${ }^{a}$ and Felix $\mathbf{Y u}^{a}$ \\ ${ }^{a}$ Department of Physics and Astronomy, University of California, \\ Irvine, California 92697, U.S.A. \\ ${ }^{b}$ Cavendish Laboratory, \\ J.J. Thomson Avenue, Cambridge, CB3 OHE, U.K. \\ ${ }^{c}$ Physics Department, Technion-Israel Institute of Technology, \\ Haifa 32000, Israel \\ ${ }^{d}$ Department of Particle Physics and Astrophysics, Weizmann Institute of Science, \\ Rehovot 76100, Israel \\ E-mail: sfrench@hep.phy.cam.ac.uk, iftah@techunix.technion.ac.il, \\ lester@hep.phy.cam.ac.uk, yosef.nir@weizmann.ac.il, yshadmi@uci.edu, \\ dsanford@uci.edu, felixy@uci.edu
}

ABSTRACT: Flavor physics may help us understand theories beyond the standard model. In the context of supersymmetry, if we can measure the masses and mixings of sleptons and squarks, we may learn something about supersymmetry and supersymmetry breaking. Here we consider a hybrid gauge-gravity supersymmetric model in which the observed masses and mixings of the standard model leptons are explained by a $\mathrm{U}(1) \times \mathrm{U}(1)$ flavor symmetry. In the supersymmetric sector, the charged sleptons have reasonably large flavor mixings, and the lightest is metastable. As a result, supersymmetric events are characterized not by missing energy, but by heavy metastable charged particles. Many supersymmetric events are therefore fully reconstructible, and we can reconstruct most of the charged sleptons by working up the long supersymmetric decay chains. We obtain promising results for both masses and mixings, and conclude that, given a favorable model, precise measurements at the LHC may help shed light not only on new physics, but also on the standard model flavor parameters.

Keywords: Supersymmetry Phenomenology

ARXIV EPRINT: 0910.1618 


\section{Contents}

1 Introduction 1

2 The model 3

$\begin{array}{llr}3 & \text { Event generation } & 6\end{array}$

4 Analysis $\quad 7$

4.1 Reconstructing the slepton NLSP $\tilde{\ell}_{1} \quad 7$

4.2 Why it is impossible to directly reconstruct $\tilde{\ell}_{2,3} \quad 9$

4.3 Reconstructing the lightest neutralino $\chi_{1}^{0}$ and $\tilde{\ell}_{2} \quad 9$

4.3.1 Direct and OS-indirect $\chi_{1}^{0}$ decays: measuring the $\chi_{1}^{0}$ and $\tilde{\ell}_{2}$ masses $\quad 10$

4.3.2 SS indirect $\chi_{1}^{0}$ decays: a clean measurement of the $\tilde{\ell}_{2}$ mass 11

4.4 Indirectly reconstructing the remaining light slepton $\tilde{\ell}_{3} \quad 12$

$\begin{array}{lll}4.5 & \text { Reconstructing the heavy sleptons } \tilde{\ell}_{4,5,6} & 12\end{array}$

$\begin{array}{lll}4.6 & \text { Reconstructing the heavier neutralino } \chi_{2}^{0} & 15\end{array}$

5 Results $\quad \mathbf{1 5}$

5.1 The light sleptons $\tilde{\ell}_{1,2,3}$ and the lightest neutralino $\chi_{1}^{0} \quad 15$

$\begin{array}{ll}5.2 \text { The heavy sleptons } \tilde{\ell}_{4,5,6} & 16\end{array}$

$\begin{array}{llr}6 & \text { Discussion } & 17\end{array}$

$\begin{array}{ll}\text { 6.1 Mass measurements } & 17\end{array}$

$\begin{array}{lll}6.2 \text { Mixings } & 18\end{array}$

$\begin{array}{lll}\text { 6.2.1 Light sleptons } & 18\end{array}$

$\begin{array}{lll}6.2 .2 & \text { Heavy sleptons } & 19\end{array}$

$\begin{array}{llr}7 & \text { Conclusions } & 19\end{array}$

A Model details $\quad 21$

$\begin{array}{lll}\text { A.1 Input parameters } & 21\end{array}$

$\begin{array}{lll}\text { A.2 Spectrum } & 22\end{array}$

\section{Introduction}

The study of flavor physics is interesting both because we do not understand why there is smallness and hierarchy in the standard model (SM) flavor parameters, and because flavor physics holds a key to understanding theories beyond the SM. If supersymmetry is discovered at the Large Hadron Collider (LHC), then studying its flavor properties - the masses and mixings of sleptons and squarks - may indeed take us a long way toward understanding the SM flavor parameters, and will also shed light on the underlying structure 
of supersymmetry and supersymmetry breaking. In this work, we take some new steps toward a quantitative analysis of the actual prospects for supersymmetric flavor measurements at the LHC, following earlier studies of lepton flavor violation at the LHC [1-9]. Our goal is to answer the following questions:

- How many sleptons might the LHC be able to identify?

- How precisely can the masses of these sleptons be measured?

- Can the flavor decomposition of these sleptons be determined?

The answers to these questions may ultimately allow us to identify the correct theory of lepton flavor, which has eluded us for decades despite plentiful experimental data. Still, even these first questions are ambitious, and for this reason our objective here is not to undertake a comprehensive study of all supersymmetric models, but rather to work toward an existence proof that, in some well-motivated cases, significant progress is possible. To do this, we will choose a favorable model to analyze.

In ref. [10], hybrid supersymmetric models were constructed in which sfermion masses receive both flavor-conserving gauge-mediated contributions and flavor-violating gravitymediated contributions governed by a $\mathrm{U}(1) \times \mathrm{U}(1)$ horizontal symmetry. These models satisfy all low-energy constraints obtained from flavor factories, and also explain all charged lepton and neutrino masses and mixings in terms of a few charge assignments under the flavor-symmetry. At the same time, the gravity-mediated effects, although subdominant, are larger than Yukawa-generated renormalization group (RG) effects and predict significant flavor mixing for sleptons and sneutrinos, with potentially striking implications for supersymmetric signals at the LHC. In these models, the lightest supersymmetric particle (LSP) is the gravitino, and the next-to-lightest supersymmetric particle (NLSP) is metastable. We will examine a model in which the NLSP is a charged slepton, the slepton mass splittings are large enough to be observable, and some flavor mixings are significant. Although we focus on this model, the techniques developed are also applicable to models with other forms of hybrid supersymmetry breaking (see, e.g., refs. [11, 12]) and other similar frameworks.

With a metastable slepton NLSP, there is no missing energy associated with the NLSP, and supersymmetric decay chains are, in principle, fully reconstructible. We choose a model in which the lightest two sleptons are predominantly a selectron and a smuon. These features are favorable for our analysis. Other features of the model are not as favorable: the two lightest sleptons are quasi-degenerate, with a mass splitting of roughly $5 \mathrm{GeV}$; the mixing of these two sleptons is small; and one of the remaining sleptons is an almost pure stau, so that its decays always involve taus which are hard to reconstruct.

In this analysis, we will only use information from electrons and muons, and assume that taus are not reconstructed at all. Even with this restrictive assumption, we find that one can extract quite a lot of information, including precision measurements of five slepton masses and one mixing angle, as well as $\mathcal{O}(1)$ estimates of the remaining mixing angles. We expect that these results may be improved, perhaps significantly, in a full analysis optimized to extract information from tau events also. 


\section{The model}

We now specify the model precisely and explain its key features. In our model, sfermion masses receive dominant flavor-conserving contributions from gauge-mediated supersymmetry breaking (GMSB) and smaller, but still significant, flavor-violating contributions from gravity-mediated supersymmetry breaking. Note that the gravity-mediated contributions are generically present, since they arise from generic Kähler potential contact terms between the supersymmetry-breaking sector and the minimal supersymmetric standard model (MSSM).

The size of the gravity-mediated contributions relative to the GMSB contributions is

$$
x \sim \frac{\tilde{m}_{\mathrm{grav}}^{2}}{\tilde{m}_{\mathrm{GMSB}}^{2}} \sim \frac{1}{N_{5}}\left(\frac{\pi M_{\mathrm{m}} F_{\mathrm{grav}}}{\alpha M_{\mathrm{Pl}} F_{\mathrm{GMSB}}}\right)^{2},
$$

where $N_{5}$ is the number of $5+\overline{5}$ messengers, $M_{\mathrm{m}}$ is the messenger scale, $M_{\mathrm{Pl}}$ is the Planck scale, and $\alpha$ stands for either the $\mathrm{SU}(2)$ or U(1) fine-structure constant. $F_{\mathrm{GMSB}}$ is the supersymmetry-breaking $F$-term associated with gauge-mediation. In addition, the supersymmetry-breaking sector typically contains other $F$ terms as well, and the gravitymediated contributions are determined by $F_{\text {grav }}^{2}=F_{\text {GMSB }}^{2}+\sum F_{i}^{2}$, where $F_{i}$ are the nonGMSB $F$-terms. If these other $F_{i}$ are negligible compared to $F_{\mathrm{GMSB}},(2.1)$ reduces to

$$
x \sim \frac{\tilde{m}_{\mathrm{grav}}^{2}}{\tilde{m}_{\mathrm{GMSB}}^{2}} \sim \frac{1}{N_{5}}\left(\frac{\pi M_{\mathrm{m}}}{\alpha M_{\mathrm{Pl}}}\right)^{2} .
$$

For the gravity contributions to be significant, either $M_{\mathrm{m}}$ must be not far below $\alpha M_{\mathrm{Pl}}$, or some $F_{i}$ 's must be larger than $F_{\mathrm{GMSB}}$.

In the models we consider, we choose $x<1$, so that the lightest supersymmetric particle (LSP) is the gravitino, with mass $m_{\tilde{G}} \sim \sqrt{x} m_{\mathrm{NLSP}}$. The NLSP will be generically long-lived in these models even for a low messenger scale, since then its decays to the gravitino are suppressed by the large $F_{\text {grav }}$.

The identity of the NLSP in these models is determined by the number of messenger fields $N_{5}$. For small $N_{5}$ it is a neutralino, and for large $N_{5}$ it is a charged slepton. We would like the NLSP to be a charged slepton, with a large enough mass splitting between the lightest neutralino and the NLSP that neutralino decays to the NLSP are observable. For very high messenger scales, however, this requires a very large $N_{5}[13] .{ }^{1}$ As an alternative, we therefore choose a moderate messenger scale $M_{\mathrm{m}} \sim 10^{6} \mathrm{GeV}$, and assume additional $F_{i}$ 's so that $F_{\mathrm{GMSB}} \ll F_{\text {grav }}$.

For the gravity-mediated contributions, the constraints from low-energy flavorchanging neutral current decays, such as $\mu \rightarrow e \gamma$, imply that large slepton mixing is possible only if there is strong degeneracy, while large mass splittings require a rather precise lepton-slepton alignment. We assume that the Froggatt-Nielsen (FN) mechanism [14] governs the flavor structure of the gravity-mediated contributions [15]. As has been shown in ref. [10], there are many possible scenarios that satisfy these constraints. We are particularly interested in models where the mass splitting between slepton generations is at least a few $\mathrm{GeV}$, so that ATLAS/CMS will perhaps be able to measure it, and where at least

\footnotetext{
${ }^{1}$ See, e.g., figure 1.
} 
some slepton mass eigenstates have appreciable components of both electron and muon flavors, so that ATLAS/CMS will have the potential of observing mixing.

Model B of ref. [10] is optimal for our purposes. First, it has $x \sim 0.1$, so that the slepton mass splittings, which are generically $\sqrt{x}$ times the slepton mass, can easily be larger than $10 \mathrm{GeV}$. Second, the flavor mixing, determined by a $\mathrm{U}(1) \times \mathrm{U}(1)$ horizontal symmetry, is significant. Schematically, the $(e, \mu, \tau)$ flavor decompositions of the slepton mass eigenstates are

$$
\begin{aligned}
\tilde{\ell}_{6} & \sim\left(\lambda^{4}, 1,1\right) \\
\tilde{\ell}_{5} & \sim\left(\lambda^{4}, 1,1\right) \\
\tilde{\ell}_{4} & \sim\left(1, \lambda^{4}, \lambda^{4}\right) \\
\tilde{\ell}_{3} & \sim\left(\lambda^{4}, \lambda^{2}, 1\right) \\
\tilde{\ell}_{2} & \sim\left(\lambda^{2}, 1, \lambda^{2}\right) \\
\tilde{\ell}_{1} & \sim\left(1, \lambda^{2}, \lambda^{4}\right),
\end{aligned}
$$

where $\lambda \sim 0.2$ is the small breaking parameter of the FN symmetry, and the sleptons are ordered by their masses from $\tilde{\ell}_{6}$, the heaviest, to $\tilde{\ell}_{1}$, the lightest. ${ }^{2}$ We see that there is significant mixing in many, although not all, of the sleptons. To fully specify Model B, one must also choose $21 \mathcal{O}(1)$ coefficients, one for each independent component of the gravitymediated contributions. We use this freedom to fix the mass ordering so that the lighter sleptons are dominantly $e$ - and $\mu$-flavored, since this improves the prospects for precision mass and mixing measurements.

Our model, then, is completely specified by the input parameters for the GMSB "spine," the horizontal symmetry and charge assignments of Model B, and the $\mathcal{O}(1)$ parameters entering the gravity-mediated sfermion mass contributions. These input parameters are listed in appendix A. We then give these input parameters to SpICE [17], a computer program that determines from these parameters the masses and flavor composition of all superparticles, along with their flavor-general decay branching ratios. SPICE interfaces with Softsusy $[18,19]$ to generate the sparticle mass spectrum, and it calls SusyHit [20] to generate non-flavor violating decays. The decays generated by SPICE include all possible lepton flavor combinations for the decays of charginos, neutralinos, sleptons, and sneutrinos. All possible two-body decays are included, along with relevant three-body slepton decays $\tilde{\ell} \rightarrow \tilde{\ell} \ell \ell$ in cases where the only kinematically allowed two-body decays involve gravitinos [21]. Note that, because of flavor mixing, the sleptons and leptons in these 3-body decays can, in principle, carry any flavor. SpICE produces HeRwig [22, 23] and SUSY Les Houches Accord [24] input files, which may be used to generate collider events. In this work, we use SPICE with Softsusy 3.0.2 and Susyhit 1.3.

The masses and flavor compositions of the six sleptons are given graphically in figure 1. (Precise values for these masses and mixings, and also for the sneutrinos, are also given in tables 2 and 3.) Although SpICE properly accounts for full $6 \times 6$ slepton mixing, in

\footnotetext{
${ }^{2}$ We assume that the spurion $\lambda$ does not have an appreciable $F$-term. This may require a rather complicated FN model [16].
} 


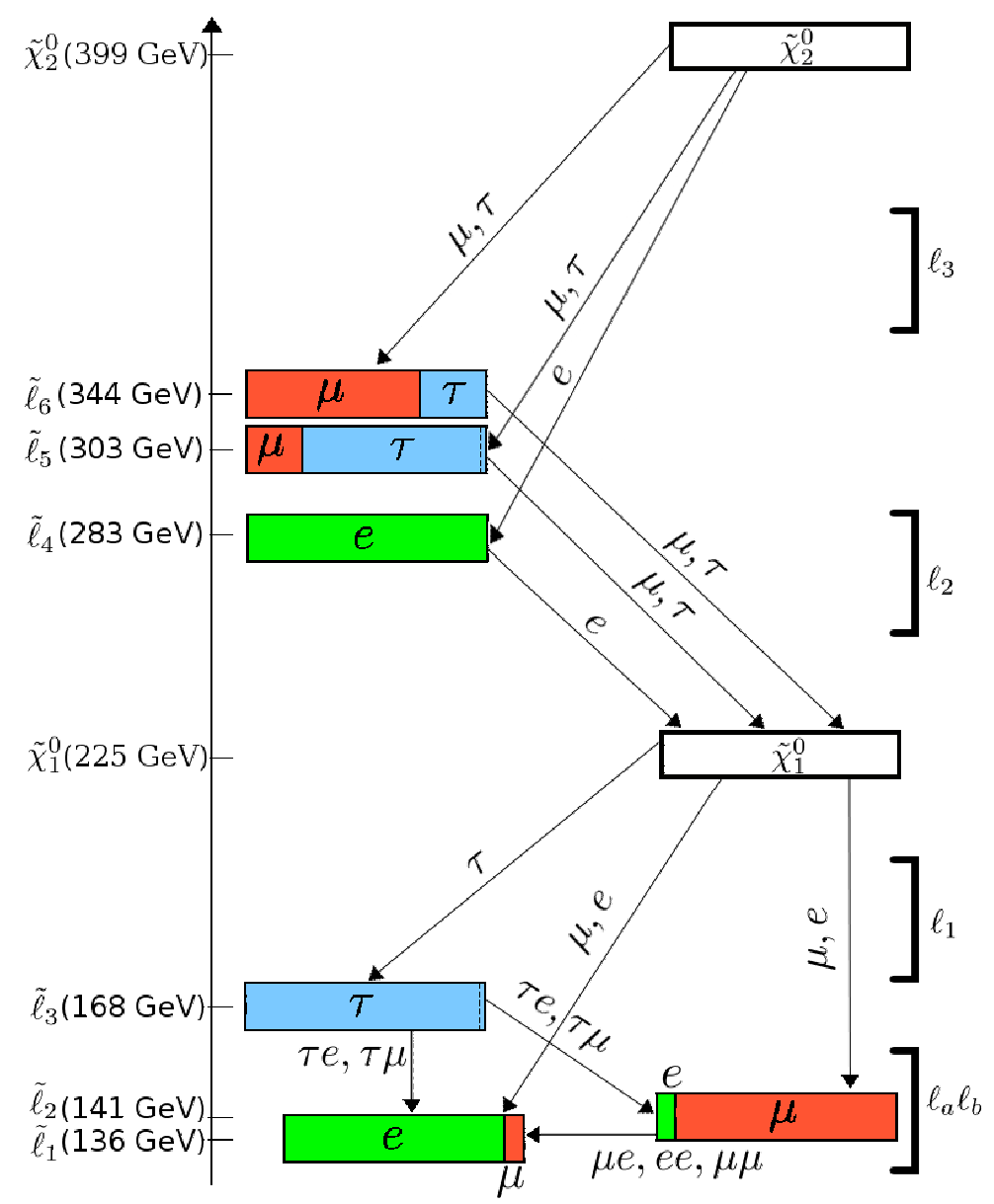

Figure 1. Slepton Mass Hierarchy. Masses and flavor compositions of the sleptons, along with the two lightest neutralinos and the leptons resulting from common decay modes. Sleptons $\tilde{\ell}_{1,2,3}$ are dominantly right-handed, with the dotted line in $\tilde{\ell}_{3}$ demarcating a small $\tilde{\tau}_{L}$ component, and $\tilde{\ell}_{4,5,6}$ are dominantly left-handed, with the dotted line in $\tilde{\ell}_{5}$ demarcating a small $\tilde{\tau}_{R}$ component. Left-right mixing in the other sleptons is negligible. The symbols $\ell_{1,2,3}$ and $\ell_{a, b}$ are used to refer to leptons produced at particular steps in the decay chain, regardless of their flavor.

this model there are no $A$-term contributions associated with the gravity-mediated contributions, and so the only left-right mixing is small and originates from RG evolution. Thus, the lighter 3 sleptons are dominantly right-handed and the heavier 3 sleptons are dominantly left-handed, with flavor compositions consistent with (2.3). Left-right mixing is small, and limited to the stau-dominated states $\tilde{\ell}_{3}$ and $\tilde{\ell}_{5}$. As desired, $\tilde{\ell}_{1}$ and $\tilde{\ell}_{2}$ have some $\tilde{e}_{R}-\tilde{\mu}_{R}$ mixing and $\tilde{\tau}_{R}$ is confined primarily to $\tilde{\ell}_{3}$. The state $\tilde{\ell}_{4}$ is dominantly $\tilde{e}_{L}$, while $\tilde{\ell}_{5,6}$ demonstrate $\mathcal{O}(1) \tilde{\mu}_{L}-\tilde{\tau}_{L}$ mixing.

As shown in figure 1, the lowest lying supersymmetric states have the following hierarchy:

$$
m_{\tilde{G}} \ll m_{\tilde{\ell}_{1,2,3}}<m_{\chi_{1}^{0}}<m_{\tilde{\ell}_{4,5,6}}<m_{\chi_{2}^{0}} .
$$

Here $\chi_{1}^{0}$ is the lightest neutralino, which is mostly the Bino, and $\chi_{2}^{0}$ is the second-lightest 
neutralino, which is, to a good approximation, the neutral Wino. The dominant decay modes are

$$
\begin{aligned}
\chi_{2}^{0} & \rightarrow \ell_{3}^{ \pm} \tilde{\ell}_{4,5,6}^{\mp} \\
\tilde{\ell}_{4,5,6}^{ \pm} & \rightarrow \ell_{2}^{ \pm} \chi_{1}^{0} \\
\chi_{1}^{0} & \rightarrow \ell_{1}^{ \pm} \tilde{\ell}_{1,2,3}^{\mp} \\
\tilde{\ell}_{3}^{ \pm} & \rightarrow \ell_{a}^{ \pm} \ell_{b}^{\mp} \tilde{\ell}_{1,2}^{ \pm}, \ell_{a}^{ \pm} \ell_{b}^{ \pm} \tilde{\ell}_{1,2}^{\mp} \\
\tilde{\ell}_{2}^{ \pm} & \rightarrow \ell_{a}^{ \pm} \ell_{b}^{\mp} \tilde{\ell}_{1}^{ \pm}, \ell_{a}^{ \pm} \ell_{b}^{ \pm} \tilde{\ell}_{1}^{\mp} .
\end{aligned}
$$

These are also shown in figure 1 . There are several noteworthy features. First, the decays of $\chi_{2}^{0}$ directly to the light sleptons $\tilde{\ell}_{1,2,3}$ are highly suppressed, since these sleptons are dominantly right-handed and have no couplings to Winos. Second, the leptons in the $\tilde{\ell}_{2,3}$ decays are quite soft and may be difficult to detect. Finally, the slepton-to-slepton $\tilde{\ell}_{2,3}$ decays may be either charge-preserving or charge-flipping. The charge-flipping modes are possible because the neutralino is a Majorana fermion, and the possibility of both kinds of decays will play a crucial role in the analysis described below.

\section{Event generation}

We generate our signal events for the LHC with center-of-mass energy $\sqrt{s}=14 \mathrm{TeV}$ using HERWIG $[22,23]$ and pass them through a generic LHC detector simulation, ACERDET 1.0 [25]. We configure ACERDET as follows: electrons and muons are selected with $p_{T}>$ $6 \mathrm{GeV}$ and $|\eta|<2.5$. Electrons and muons are considered to be isolated if they are separated from other leptons and jets by $\Delta R>0.4$, where $\Delta R=\sqrt{(\Delta \eta)^{2}+(\Delta \phi)^{2}}$, and if less than $10 \mathrm{GeV}$ of energy is deposited in a cone of $\Delta R=0.2$. The lepton momentum resolutions we use are parameterized from the results of Full Simulation of the ATLAS detector [26]; our electrons are smeared according to a pseudorapidity-dependent parametrization, while muons are smeared according to the results for $|\eta|<1.1$. The leading-order two-to-two supersymmetric cross section determined by HERWIG with CTEQ5L parton distribution functions is $1.154 \mathrm{pb}$. We generate 115,400 events, corresponding to $100 \mathrm{fb}^{-1}$ of data. ACERDET does not take into account lepton reconstruction efficiencies. We therefore apply by hand a reconstruction efficiency of $90 \%$ to the muons and a reconstruction efficiency of $77 \%$ to the electrons. This gives 0.86 as the ratio of electron to muon reconstruction efficiency.

In a real detector or full simulation thereof, a long-lived charged slepton, such as our $\tilde{\ell}_{1}$, would be expected to produce a visible track by virtue of its charge. Measurement of the curvature of this track would determine the $\tilde{\ell}_{1}$ momentum. ${ }^{3}$ If the $\tilde{\ell}_{1}$ arrival time were also to be measured with sufficient accuracy, the $\tilde{\ell}_{1}$ speed could also be determined. The momentum and speed may be combined to determine the track mass, which will constitute the primary signature for the existence of new physics (i.e., the $\tilde{\ell}_{1}$ ) in our signal events. ACERDET is not a full detector simulation and so, unsurprisingly, does not produce track objects or speed "measurements" associated with our long-lived sleptons.

\footnotetext{
${ }^{3}$ Strictly speaking, the curvature determines only the momentum-to-charge ratio, but we will assume unit charges for all heavy charged tracks.
} 
Since measurement errors associated with the $\tilde{\ell}_{1}$ are potentially very important in our study, it is necessary to augment ACERDET by incorporating additional parameterizations

for $\tilde{\ell}_{1}$ momentum and speed measurements. We used the resolutions taken from ref. [27], based on ATLAS Muon Resistive Plate Chamber (RPC) timings. Given these results, to model the reconstructed $\tilde{\ell}_{1}$ momentum, we start with the momentum taken from Monte Carlo truth, and then smear the slepton's 3-momentum magnitudes $p \equiv\left|\vec{p}_{\tilde{\ell}_{1}}\right|$ and speeds $\beta$ by Gaussian distributions with $\sigma_{p} / p=0.05$ and $\sigma_{\beta}=0.02$, respectively. The slepton's energy and direction are not smeared. Note that we could just as easily have used another parametrization based on ATLAS Muon Drift Tube (MDT) fits [28]. Our use of just one such source of timing information might therefore mean that our speed resolution can be improved in a future study. We only consider sleptons with $\beta>0.6$ to be reconstructible, because sleptons moving any slower are unlikely to reach the muon chambers quickly enough to be registered in the same bunch crossing. In our events, $95 \%$ of sleptons have a speed, $\beta$, greater than 0.6 .

Having parametrized the slepton reconstruction process above, subsequent uses of expressions like " $\tilde{l}_{1}$ momentum" and " $\beta$ " refer to the smeared (supposedly reconstructed) quantities rather than to Monte Carlo truth.

\section{Analysis}

In this section we outline our approach to measuring the mixings and masses of the six sleptons. As our NLSP is a metastable charged particle, our entire decay chain is, in principle, fully reconstructible. Our goal is therefore to reconstruct the various superpartners, starting with the slepton NLSP, by working our way up the decay chain, constructing various invariant mass distributions. In this section we explain each invariant mass distribution and in the next section we interpret the results in terms of mixings and masses.

As explained in section 1, in this analysis we ignore tau leptons, and instead only consider what we can deduce about masses and mixings from observing electrons and muons. We make this choice because tau reconstruction is expected to be poor in comparison to electron and muon reconstruction, but the inclusion of tau reconstruction certainly merits further study.

\subsection{Reconstructing the slepton NLSP $\tilde{\ell}_{1}$}

In each event, direct reconstruction of the $\tilde{\ell}_{1}$ momentum, speed, and mass is expected to be relatively straightforward, using the slow charged track signature already described in section 3. We restrict our attention to sleptons with speeds in the range $0.6<\beta<0.8$. The necessity of the lower bound has already been mentioned. The upper bound effectively distinguishes supersymmetric events from background [29-32], and also plays a crucial role in improving the resolution of the $\tilde{\ell}_{1}$ mass measurement. Of the sleptons with $\beta>0.6$, $15 \%$ have $\beta<0.8$ and so pass this extra restriction. The reconstructed mass distribution is shown in figure 2 before and after we impose the upper $\beta$ limit. As expected, even ignoring background that will enter the $\beta>0.6$ distribution (but is not included in figure $2 \mathrm{a}$ ), this distribution is fit poorly by a Gaussian curve, and hence we obtain a better measurement 


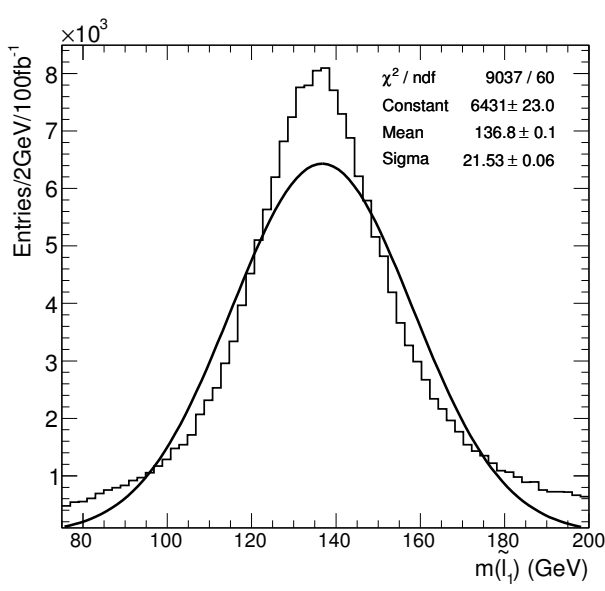

(a) $\beta>0.6$

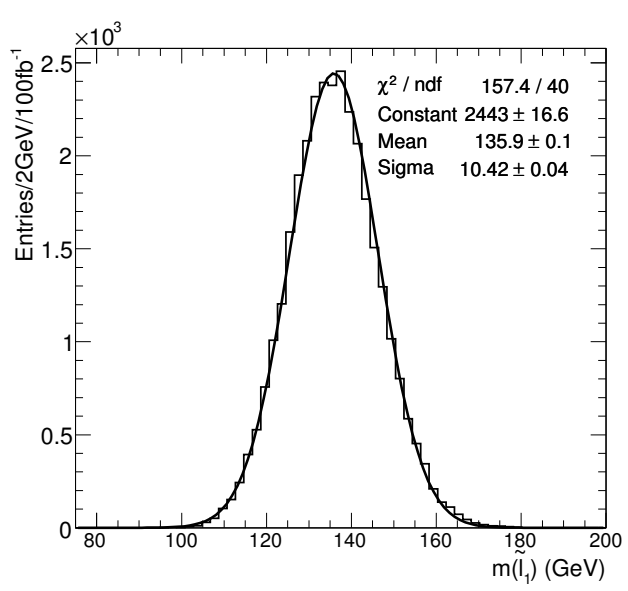

(b) $0.6<\beta<0.8$

Figure 2. Reconstructed $\tilde{\ell}_{1}$ Mass Distributions. Reconstructed $\tilde{\ell}_{1}$ masses from events with slepton speed (a) $\beta>0.6$ and (b) $0.6<\beta<0.8$. The histograms are the distributions, and the solid lines are Gaussian fits with means and standard deviations as indicated. The Gaussian fit for (a) is poor and is shown for comparison purposes only, as described in the text. The $\tilde{\ell}_{1}$ 's 3-momentum magnitudes $p \equiv\left|\vec{p}_{\tilde{\ell}_{1}}\right|$ and speeds have been smeared by Gaussian distributions with $\sigma_{p} / p=0.05$ and $\sigma_{\beta}=0.02$, respectively.

of slepton mass by considering the slowest of the sleptons. After requiring $0.6<\beta<0.8$, we assume that the efficiency for reconstructing $\tilde{\ell}_{1}$ is $100 \%$, and we do not require these sleptons to pass any isolation criteria.

Figure 2 tells us two valuable things. First, for any given event, the slepton mass is measured to an accuracy of the order of $10 \mathrm{GeV}$. Second, from taking all of the events together, the statistical error on the mean mass is much smaller, of the order of $0.1 \mathrm{GeV}$. The strong agreement between the underlying $\tilde{\ell}_{1}$ mass and the fitted mean reconstructed $\tilde{\ell}_{1}$ mass in figure 2 is perhaps misleading. Such agreement was to be expected as no sources of systematic offsets were introduced in our smearing process. Realistically, the fitted mean reconstructed $\tilde{\ell}_{1}$ mass will be subject to some systematic offset, even after all attempts to calibrate the detector have been completed. The size and direction of such an offset cannot be known in advance, so we do not attempt to simulate any systematic offset in this analysis.

We can make use of the high precision (mean) mass measurement from the set of all events to help us remove some of the momentum and time-of-flight measurement errors in individual events, thereby reducing our exposure to the $10 \mathrm{GeV}$ event-by-event variability in reconstructed slepton masses. We do this by scaling each component of the four-momentum of every slow charged track by a constant so that the track mass matches the mean mass obtained from the fit to all events. After rescaling, the smeared and rescaled momentum is centered on the true momentum with a full-width half-maximum of $27 \mathrm{GeV}$ and $\mathrm{rms}$ of $9.9 \mathrm{GeV}$, and the corresponding energy difference distribution has a full-width halfmaximum of $31 \mathrm{GeV}$ and $\mathrm{rms}$ of $9.4 \mathrm{GeV}$. We find that this event-by-event rescaling process is necessary to allow us sufficient resolution to travel up the decay chain and determine the masses of sparticles heavier than the NLSP. 


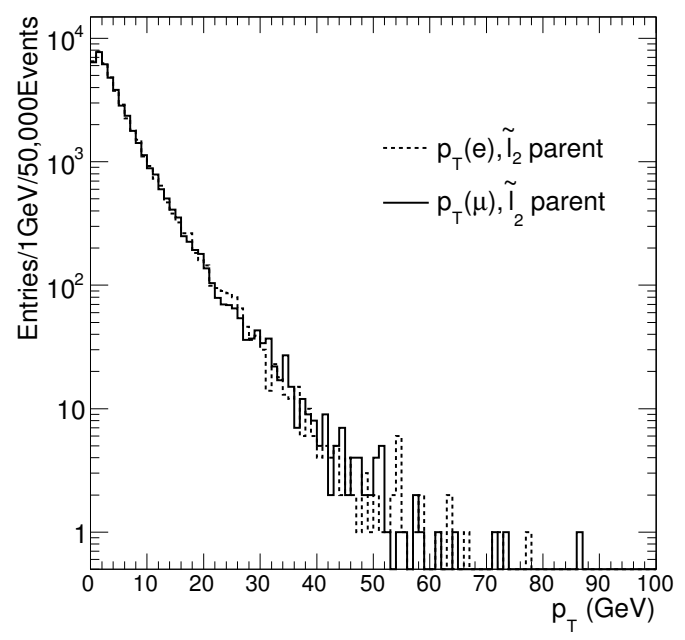

Figure 3. Three Body $p_{T}$ Distribution. The $p_{T}$ distribution of leptons $\ell=e, \mu$ from the three body decays $\tilde{\ell}_{2} \rightarrow \ell \ell \tilde{\ell}_{1}$ from Monte Carlo truth.

\subsection{Why it is impossible to directly reconstruct $\tilde{\ell}_{2,3}$}

The next most obvious particles to reconstruct are the next two lightest sleptons, $\tilde{\ell}_{2,3}$. We see in figure 1 that the dominant $\tilde{\ell}_{2,3}$ decays are three-body. In principle, given an ideal detector, we could reconstruct the $\tilde{\ell}_{2,3}$ by looking at the three-particle invariant mass distributions resulting from combining the $\tilde{\ell}_{1}$ with all possible combinations of two further leptons which give a charged $\tilde{\ell}_{2,3}$ candidate.

Since in this analysis we are not reconstructing taus, we cannot detect $\tilde{\ell}_{3}$ in this way. We will briefly comment on the $\tilde{\ell}_{3}$ in section 4.4 .

Unfortunately, direct reconstruction of $\tilde{\ell}_{2}$ is also impossible as $\tilde{\ell}_{1}$ and $\tilde{\ell}_{2}$ are nearly degenerate. In figure 3 we plot the true $p_{T}$ of all leptons produced in the three-body $\tilde{\ell}_{2}$ decays. We see that $\sim 90 \%$ of all electrons and muons have $p_{T}<10 \mathrm{GeV}$. Leptons with $p_{T}<10 \mathrm{GeV}$, which we denote "soft" leptons, will be very difficult to reconstruct in ATLAS/CMS.

To determine the mass and mixings of the $\tilde{\ell}_{2}$, we must therefore rely on indirect measurements. These do, in fact, make a measurement of the $\tilde{\ell}_{2}$ mass possible, as we describe in the next section.

\subsection{Reconstructing the lightest neutralino $\chi_{1}^{0}$ and $\tilde{\ell}_{2}$}

In this section we describe the $\chi_{1}^{0}$ reconstruction, and explain how it can be used to detect $\tilde{\ell}_{2}$ and measure its mass as well.

Referring to figure 1 , we see that the neutralino can decay to any one of the three light sleptons. Let us first discuss neutralino decays to $\tilde{\ell}_{1}$ and $\tilde{\ell}_{2}$, with the $\tilde{\ell}_{2}$ subsequently decaying to $\tilde{\ell}_{1}$ via a 3 -body decay. Since the leptons involved in this 3 -body decay, $\ell_{a, b}$, are typically soft, they will usually go undetected, so that one only observes the final $\tilde{\ell}_{1}$ 
and the hard lepton, $\ell_{1}$, coming from the original neutralino decay. (Throughout this section, subscripts on the leptons are as shown in figure 1.) We therefore define two main categories of neutralino decay - direct and indirect. The latter is further divided into two subcategories, which we name "OS indirect" and "SS indirect," based on whether the final $\tilde{\ell}_{1}$ has the opposite or same sign as the lepton $\ell_{1}$ :

- Direct decays of $\chi_{1}^{0}$ to the slepton NLSP: $\chi_{1}^{0} \rightarrow \tilde{\ell}_{1}^{ \pm} \ell_{1}^{\mp}$

- Indirect decays of the $\chi_{1}^{0}$ to the slepton NLSP via $\tilde{\ell}_{2}, \chi_{1}^{0} \rightarrow \tilde{\ell}_{2}^{ \pm} \ell_{1}^{\mp}$, followed by a three-body $\tilde{\ell}_{2}$ decay of one of the following types:

$$
\begin{array}{lll}
\circ & \tilde{\ell}_{2}^{ \pm} \rightarrow \tilde{\ell}_{1}^{ \pm} \ell_{a}^{ \pm} \ell_{b}^{\mp} & \text { OS indirect } \\
\circ & \tilde{\ell}_{2}^{ \pm} \rightarrow \tilde{\ell}_{1}^{\mp} \ell_{a}^{ \pm} \ell_{b}^{ \pm} & \text {SS indirect }
\end{array}
$$

Note that in the instance of a direct decay, the $\tilde{\ell}_{1}$ and the lepton $\ell_{1}$ must be oppositely charged, whereas in indirect decays, same-sign charges are also possible. Detection of samesign events are direct evidence of the Majorana nature of the neutralino. As mentioned above, with a hard lepton $p_{T}$ cut, only the lepton $\ell_{1}$ is observed.

\subsubsection{Direct and OS-indirect $\chi_{1}^{0}$ decays: measuring the $\chi_{1}^{0}$ and $\tilde{\ell}_{2}$ masses}

For both the direct and OS indirect decays, the $\tilde{\ell}_{1}$ and the lepton $\ell_{1}$ have opposite signs. These channels therefore have identical signatures and are therefore reconstructed in tandem. We take all OS $\tilde{\ell}_{1}^{ \pm} e^{\mp}$ and $\tilde{\ell}_{1}^{ \pm} \mu^{\mp}$ combinations and reconstruct the invariant masses $m_{\tilde{\ell}_{1}^{ \pm} e^{\mp}}$ and $m_{\tilde{\ell}_{1}^{ \pm} \mu^{\mp}}$.

When our OS $\tilde{\ell}_{1}$ and lepton are from a direct decay, we expect to reconstruct the $\chi_{1}^{0}$ mass exactly. However, when the $\tilde{\ell}_{1}$ results from an indirect decay, we do not expect to correctly reconstruct the $\chi_{1}^{0}$ as we are missing the two soft leptons produced in the decay $\tilde{\ell}_{2}^{ \pm} \rightarrow \tilde{\ell}_{1}^{ \pm} \ell_{a}^{+} \ell_{b}^{-}$. Such events nevertheless contain valuable information: as shown in ref. [33], the $\tilde{\ell}_{1}$-lepton invariant mass then exhibits a "shifted peak," somewhat lower than the neutralino mass, by an amount

$$
E_{\text {shift }} \simeq \frac{M^{2}+m_{1}^{2}}{2 M m_{1}} \Delta m
$$

Here $M$ is the neutralino mass, the mean of the $\tilde{\ell}_{1} \ell_{1}$ invariant mass distribution, $m_{1}$ is the reconstructed mean of the $\tilde{\ell}_{1}$ mass, and $\Delta m \equiv m_{\tilde{\ell}_{2}}-m_{\tilde{\ell}_{1}}$. For our model parameters we predict $E_{\text {shift }} \simeq 5.6 \mathrm{GeV}$. By measuring this shift we can deduce $\Delta m$ and determine the mass of the $\tilde{\ell}_{2}$ indirectly.

We plot these OS slepton-lepton invariant mass distributions in figure 4 for both $p_{T}>10 \mathrm{GeV}$ and $p_{T}>30 \mathrm{GeV}$. The harder $p_{T}$ cut effectively removes the soft leptons produced in three-body $\tilde{\ell}_{2,3}$ decays that have not already failed reconstruction from the lepton collections (see figure 3). We decompose these distributions (and the distributions that follow) into the sum of an exponentially falling background and one or more Gaussian peaks. The exponentially falling background is designed to model the combinatoric 


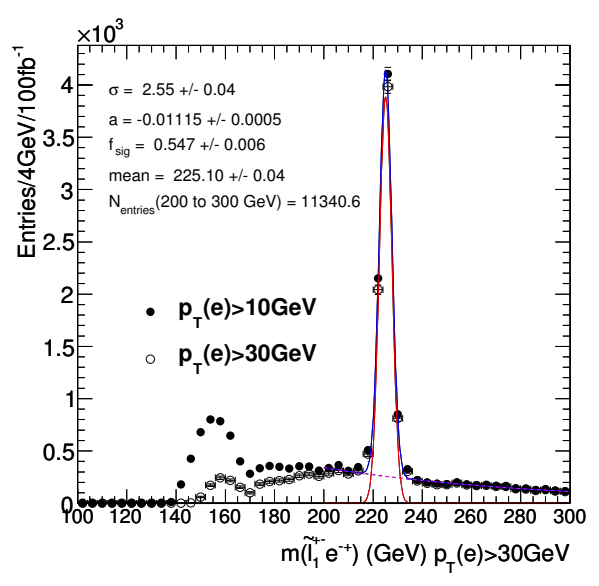

(a) $\ell=e$

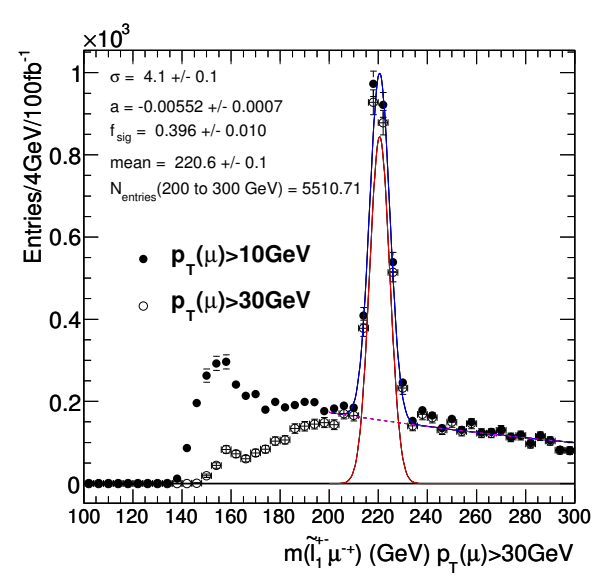

(b) $\ell=\mu$

Figure 4. OS $\tilde{\ell}_{1}^{ \pm} \ell^{\mp}$ Invariant Mass Distributions. Invariant mass distributions of OS $\tilde{\ell}_{1}^{ \pm} \ell^{\mp}$ pairs for (a) $\ell=e$ and (b) $\ell=\mu$, requiring $p_{T}>10 \mathrm{GeV}$ and $p_{T}>30 \mathrm{GeV}$. The $p_{T}>30 \mathrm{GeV}$ distributions in the range $200 \mathrm{GeV}<m<300 \mathrm{GeV}$ have been fit with a Gaussian peak on top of an exponentially decaying background as given by the red (lighter) solid and purple (lighter) dashed contours, respectively. The sum of these fits is given by the blue (darker) solid line. The fit parameters, defined in (4.2), are as indicated.

background from supersymmetric events. The fitting function has the form

$$
\frac{d N}{d m}=N_{\text {tot }}\left[\left(1-\sum_{i} f_{i}\right)\left(-a_{i}\right) e^{a_{i} m}+\sum_{i} f_{i} \sqrt{\frac{2}{\pi}} \frac{1}{\sigma_{i}} e^{-\frac{\left(m-\bar{m}_{i}\right)^{2}}{2 \sigma_{i}^{2}}}\right],
$$

where $N_{\text {tot }}$ is the total number of events in the distribution, $a_{i}$ is the exponential decay parameter with units of $\mathrm{GeV}^{-1}, f_{i}$ is the fraction of the total number of events in peak $i$, and $\bar{m}_{i}$ and $\sigma_{i}$ are the center and width of peak $i$.

Indeed, we see two different peaks: a higher one at $225.1 \mathrm{GeV}$ in the $\tilde{\ell}_{1}^{ \pm} e^{\mp}$ sample, corresponding to the true neutralino mass, and the lower shifted peak centered at $220.6 \mathrm{GeV}$ in the $\tilde{\ell}_{1}^{ \pm} \mu^{\mp}$ sample. Of course, in the model we are considering, each one of these samples contains both these peaks, but because of the flavor compositions of $\tilde{\ell}_{1}$ and $\tilde{\ell}_{2}$, the $\tilde{\ell}_{1}^{ \pm} e^{\mp}$ sample is dominated by direct decays, and therefore seems to exhibit just the unshifted peak at $\sim 225 \mathrm{GeV}$, while the $\tilde{\ell}_{1}^{ \pm} \mu^{\mp}$ sample is dominated by indirect decays, and therefore seems to exhibit just the shifted peak at $\sim 220 \mathrm{GeV}$. In a general model, with larger mixings, one would see a double-peak structure, which might be harder to disentangle. Then, however, the SS decays can come to our aid: these can only originate from indirect decays, and thus will cleanly exhibit only the shifted peak, with no contamination from direct decays.

\subsubsection{SS indirect $\chi_{1}^{0}$ decays: a clean measurement of the $\tilde{\ell}_{2}$ mass}

As explained above, the SS $\tilde{\ell}_{1}^{ \pm} \ell^{ \pm}$sample cleanly probes the indirect neutralino decays through $\tilde{\ell}_{2}$, and exhibits just the neutralino shifted peak. Here we take all SS $\tilde{\ell}_{1}^{ \pm} e^{ \pm}$and 


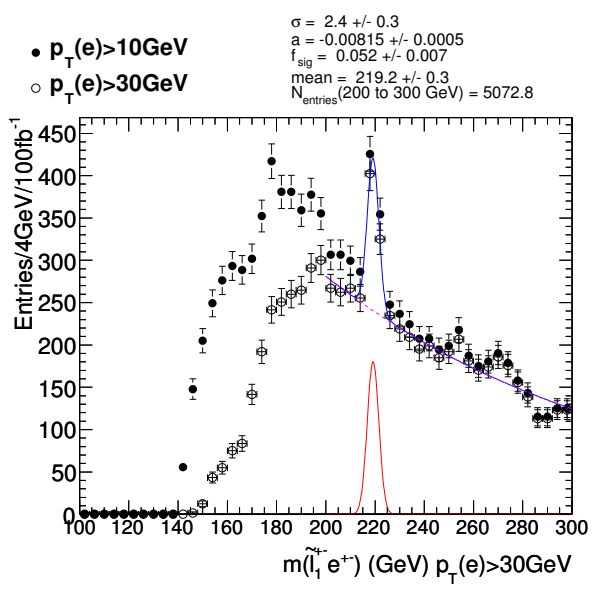

(a) $\ell=e$

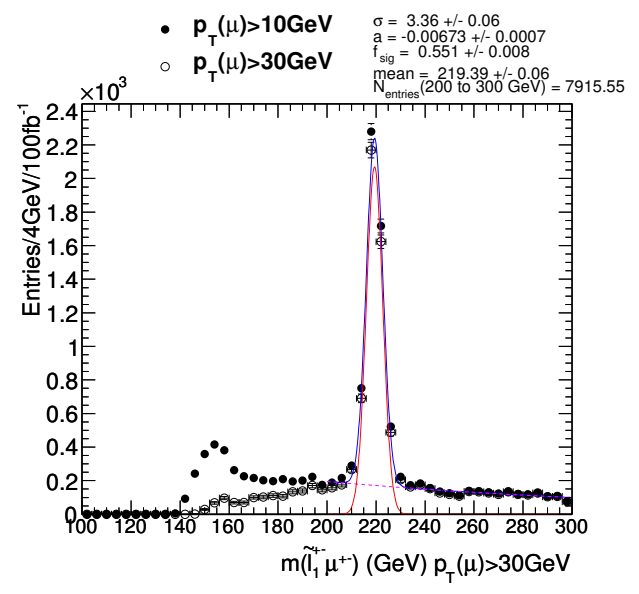

(b) $\ell=\mu$

Figure 5. $S S \tilde{\ell}_{1}^{ \pm} \ell^{ \pm}$Invariant Mass Distributions. As in figure 4 , but for SS $\tilde{\ell}_{1}^{ \pm} \ell^{ \pm}$pairs.

$\tilde{\ell}_{1}^{ \pm} \mu^{ \pm}$combinations and reconstruct the invariant masses $m_{\tilde{\ell}_{1}^{ \pm} e^{ \pm}}$and $m_{\tilde{\ell}_{1}^{ \pm} \mu^{ \pm}}$. Again we do so for both $p_{T}>10 \mathrm{GeV}$ and $p_{T}>30 \mathrm{GeV}$. The invariant mass distributions are shown in figure 5, and indeed, we only see the neutralino shifted peak at around $219 \mathrm{GeV}$. It is not surprising that the peaks in these distributions are somewhat lower than the shifted peak of figure $4 \mathrm{~b}$. The SS samples necessarily come from neutralino decays through $\tilde{\ell}_{2}$, which only exhibit the shifted peak, whereas the OS $\tilde{\ell}_{1} \mu$ sample, although dominated by such decays, also contains some events in which the neutralino decays directly to $\tilde{\ell}_{1}$. The latter lead to the correct neutralino peak at $225 \mathrm{GeV}$.

\subsection{Indirectly reconstructing the remaining light slepton $\tilde{\ell}_{3}$}

Inspecting figures 4 and 5 also reveals a smaller peak around $160 \mathrm{GeV}$. It is easy to see that this peak corresponds, at least partially, to $\tilde{\ell}_{3}$. Consider $\tilde{\ell}_{3}$ decays to $\tilde{\ell}_{1}$. These are dominantly $\tilde{\ell}_{3} \rightarrow \tilde{\ell}_{1} e \tau$. The tau decay could give another charged lepton, but this charged lepton would typically be softer than the original electron produced in the $\tilde{\ell}_{3}$ decay. Thus, when we consider the invariant mass of this electron paired with the $\tilde{\ell}_{1}$, we should find a peak somewhat below the $\tilde{\ell}_{3}$ mass, much like the shifted neutralino peak discussed in the previous two sections. An analogous peak should also occur for $\tilde{\ell}_{1} \mu$ pairs, coming either from direct $\tilde{\ell}_{3} \rightarrow \tilde{\ell}_{1} \mu \tau$ decays, or from indirect decays through $\tilde{\ell}_{3} \rightarrow \tilde{\ell}_{2} \mu \tau$.

However, this peak starts at around $140 \mathrm{GeV}$, which, as we already know, is the $\tilde{\ell}_{2}$ mass. We therefore expect another peak around $140 \mathrm{GeV}$, originating from $\tilde{\ell}_{2} \rightarrow \tilde{\ell}_{1}$ decays with one soft lepton. In a real detector, smearing effects would then make it hard to conclusively establish the identity of the $160 \mathrm{GeV}$ peak. We therefore leave the question of $\tilde{\ell}_{3}$ identification for future work, in which tau leptons are carefully treated.

\subsection{Reconstructing the heavy sleptons $\tilde{\ell}_{4,5,6}$}

Once the $\chi_{1}^{0}$ is reconstructed, we can use it as a base for reaching higher up the decay chain. As evident in figure 1 , the heavier sleptons dominantly decay through $\tilde{\ell}_{4,5,6} \rightarrow \chi_{1}^{0} \ell_{2}$. 


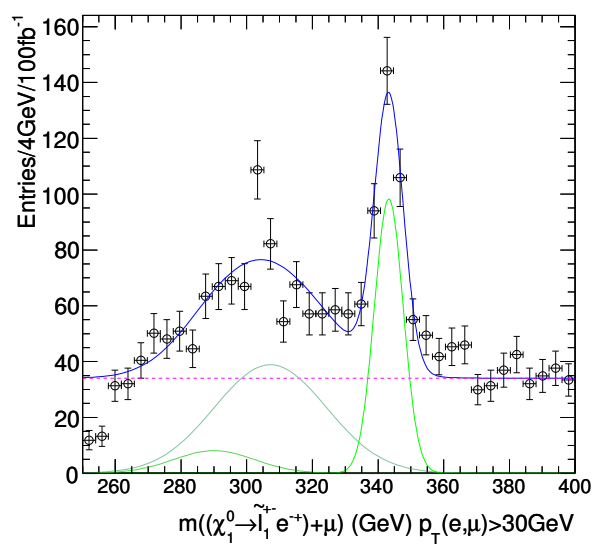

(a) $\left(\ell_{1}, \ell^{\prime}\right)=(e, \mu)$

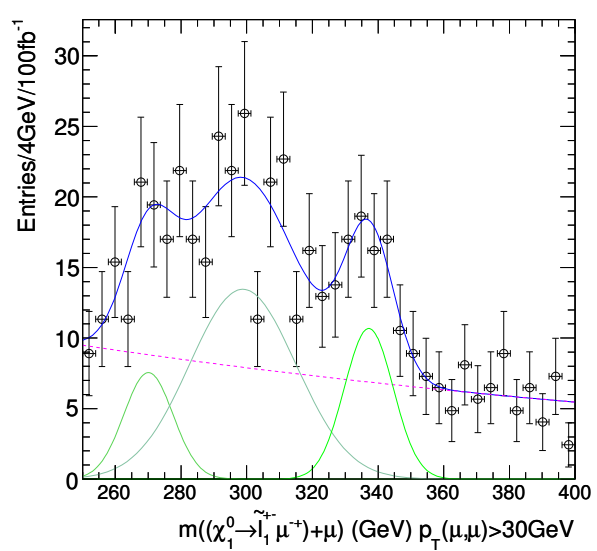

(c) $\left(\ell_{1}, \ell^{\prime}\right)=(\mu, \mu)$

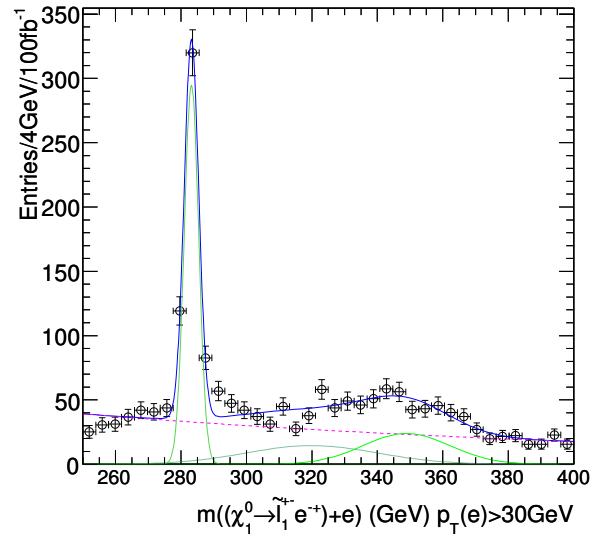

(b) $\left(\ell_{1}, \ell^{\prime}\right)=(e, e)$

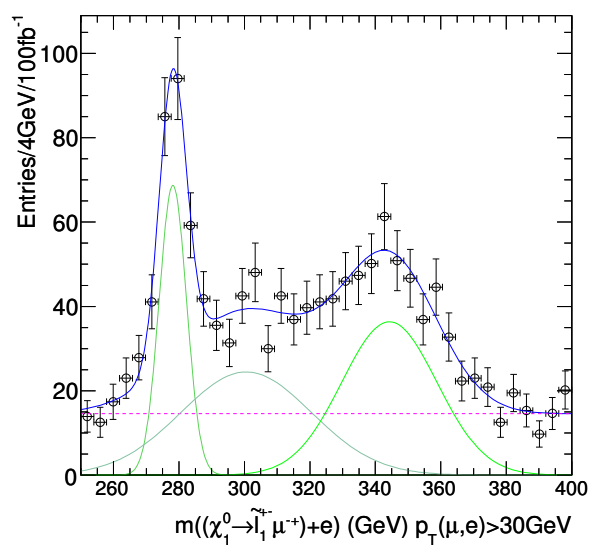

(d) $\left(\ell_{1}, \ell^{\prime}\right)=(\mu, e)$

Figure 6. OS Slepton-Dilepton Invariant Mass Distributions. Invariant mass distributions of $\left(\tilde{\ell}_{1}^{ \pm} \ell_{1}^{\mp}\right) \ell^{\prime}$, where the $\tilde{\ell}_{1}^{ \pm} \ell_{1}^{\mp}$ pair reconstructs $\chi_{1}^{0}$ as described in the text, and $\left(\ell_{1}, \ell^{\prime}\right)=(\mathrm{a})(e, \mu)$, (b) $(e, e),(\mathrm{c})(\mu, \mu)$, and (d) $(\mu, e)$. Both $\ell_{1}$ and $\ell^{\prime}$ are required to have $p_{T}>30 \mathrm{GeV}$. These distributions in the range $250 \mathrm{GeV}<m<400 \mathrm{GeV}$ have been fit with Gaussian peaks on top of an exponentially decaying background as given by the green (lighter) solid and purple (lighter) dashed contours, respectively. The sum of these fits is given by the blue (darker) solid line.

Since in our case no heavy slepton is purely stau, all three of the sleptons are in principle accessible even by reconstructing only electrons and muons.

We take all $\tilde{\ell}_{1}^{ \pm} \ell_{1}^{\mp}$ combinations lying within $2 \sigma$ of the mean of the OS $\tilde{\ell}_{1} \ell_{1}$ invariant mass distributions of figure 4 and combine them with yet another lepton $\ell^{\prime}=e, \mu$ to obtain $\left(\tilde{\ell}_{1}^{ \pm} \ell_{1}^{\mp}\right) \ell^{\prime}$ invariant mass distributions. Here and in the following we enclose $\tilde{\ell}_{1} \ell_{1}$ in parentheses to indicate a $\tilde{\ell}_{1}$-lepton pair that reconstructs the $\chi_{1}^{0}$. We require that both $\ell_{1}$ and $\ell^{\prime}$ have $p_{T}>30 \mathrm{GeV}$. This essentially gives us the $m_{\chi_{1}^{0} \ell^{\prime}}$ invariant mass distribution. These invariant mass distributions, with fitted peaks, are shown in figure 6 . 


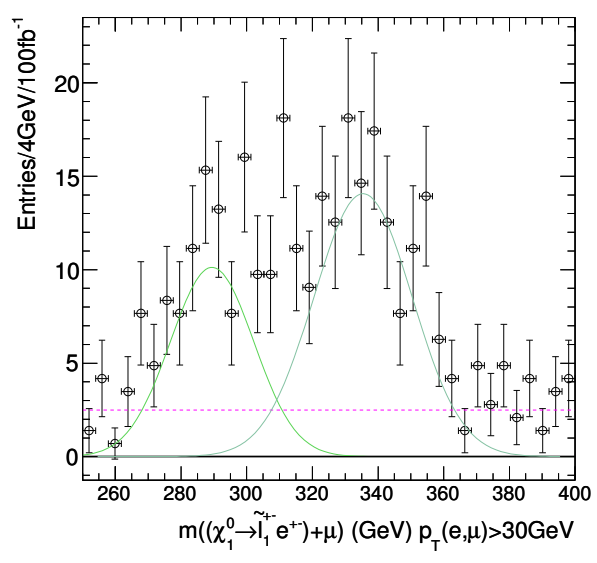

(a) $\left(\ell_{1}, \ell^{\prime}\right)=(e, \mu)$

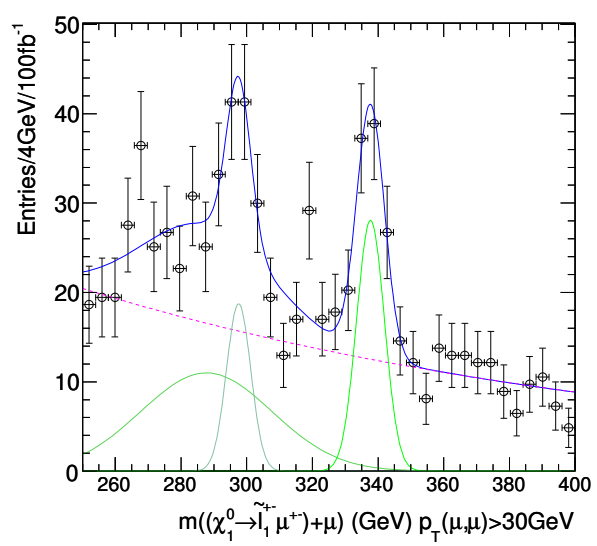

(c) $\left(\ell_{1}, \ell^{\prime}\right)=(\mu, \mu)$

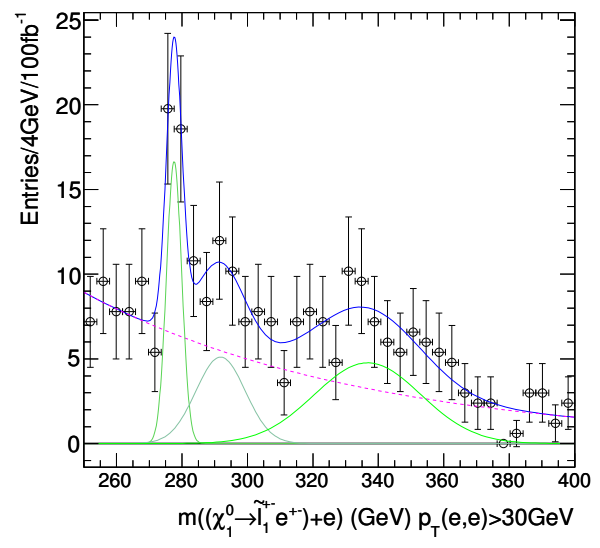

(b) $\left(\ell_{1}, \ell^{\prime}\right)=(e, e)$

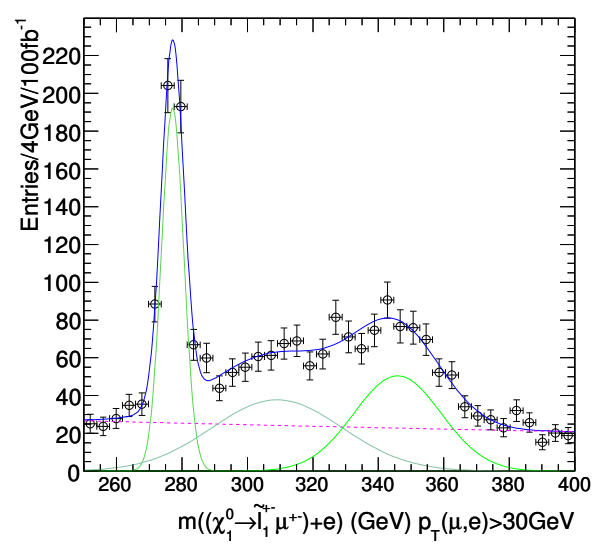

(d) $\left(\ell_{1}, \ell^{\prime}\right)=(\mu, e)$

Figure 7. SS Slepton-Dilepton Invariant Mass Distributions. As in figure 6, but for SS sleptondilepton combinations $\left(\tilde{\ell}_{1}^{+} \ell_{1}^{+}\right) \ell^{\prime \pm}$ and $\left(\tilde{\ell}_{1}^{-} \ell_{1}^{-}\right) \ell^{\prime \pm}$.

We can also take all combinations of SS $\tilde{\ell}_{1}$-lepton pairs with invariant masses falling within $2 \sigma$ of the SS primary $\tilde{\ell}_{1}$-lepton invariant mass distribution means and combine them with another lepton. The resulting invariant mass distributions, with fitted peaks, are plotted in figure 7. Again we require that both leptons have $p_{T}>30 \mathrm{GeV}$. We know that these combinations give a poorer reconstruction of $\tilde{\ell}_{4,5,6}$ than the OS combinations, since SS $\tilde{\ell}_{1} \ell_{1}$ pairs reconstruct not the neutralino mass but the neutralino shifted peak, but we include these plots for completeness. Note that there is another reason why we expect these plots to give an inferior reconstruction of the heavier sleptons compared to the OS plots of figure 6 . The combinatoric background is larger here, since it includes $\tilde{\ell}_{1}$ plus two lepton combinations with charges summing to either \pm 1 or to \pm 3 , whereas the OS plots only include the former. 


\begin{tabular}{|c|c|c|c|}
\hline & True & Measured & Observation \\
\hline$\tilde{\ell}_{1}$ & $135.83 \mathrm{GeV}$ & $135.9 \pm 0.1 \mathrm{GeV}$ & direct observation of $\tilde{\ell}_{1}$ with $0.6<\beta\left(\tilde{\ell}_{1}\right)<0.8$ (figure $\left.2 \mathrm{~b}\right)$ \\
$\chi_{1}^{0}$ & $224.83 \mathrm{GeV}$ & $225.10 \pm 0.04 \mathrm{GeV}$ & $\chi_{1}^{0}$ peak in the $\tilde{\ell}_{1}^{ \pm} e^{\mp}$ invariant mass distribution (figure 4a) \\
$\Delta m\left(\tilde{\ell}_{1,2}\right)$ & $4.95 \mathrm{GeV}$ & $5.06 \pm 0.06 \mathrm{GeV}$ & $\tilde{\ell}_{1}^{ \pm} e^{\mp}$ minus $\tilde{\ell}_{1}^{ \pm} \mu^{ \pm}$peak positions (figure 4a, figure $\left.5 \mathrm{~b}\right)$ \\
$\tilde{\ell}_{4}$ & $282.86 \mathrm{GeV}$ & $283.2 \pm 0.1 \mathrm{GeV}$ & peak in $\left(\tilde{\ell}_{1}^{\mp} e^{ \pm}\right) e$ invariant mass distribution (figure 6b) \\
$\tilde{\ell}_{5}$ & $303.41 \mathrm{GeV}$ & $307 \pm 5 \mathrm{GeV}$ & peak in $\left(\tilde{\ell}_{1}^{\mp} e^{ \pm}\right) \mu$ invariant mass distribution (figure 6a) \\
$\tilde{\ell}_{6}$ & $343.53 \mathrm{GeV}$ & $343.3 \pm 0.5 \mathrm{GeV}$ & peak in $\left(\tilde{\ell}_{1}^{\mp} e^{ \pm}\right) \mu$ invariant mass distribution (figure 6a) \\
$\left|U_{2 e} / U_{2 \mu}\right|^{2}$ & 0.069 & $0.071 \pm 0.010$ & $N\left(\tilde{\ell}_{1}^{ \pm} e^{ \pm}\right) / N\left(\tilde{\ell}_{1}^{ \pm} \mu^{ \pm}\right)$(figure 5) \\
\hline
\end{tabular}

Table 1. Results. Best measurements of slepton masses and mixings in our model. The measurement for $\tilde{\ell}_{5}$ is given under the assumption that the excess of events we see is indeed a mass resonance, and not a fluctuation of the background.

\subsection{Reconstructing the heavier neutralino $\chi_{2}^{0}$}

With every step up the chain, the reconstruction errors compound, making $\chi_{2}^{0}$ recovery harder than $\chi_{1}^{0}$. In this analysis we do not attempt to reconstruct the heavier neutralino. Nevertheless, we note that imposing the additional constraint that some lepton combinations reconstruct the $\chi_{2}^{0}$ may provide additional information and allow constraints on flavor mixings among the heavy sleptons.

\section{Results}

We now take our invariant mass distributions and interpret them in terms of slepton masses and mixings. Our best measurements of slepton masses and mixings in this model are summarized in table 1 , and the individual entries are supported by the detailed discussions below.

\subsection{The light sleptons $\tilde{\ell}_{1,2,3}$ and the lightest neutralino $\chi_{1}^{0}$}

As explained in section 4.4 we do not attempt to measure the $\tilde{\ell}_{3}$ mass in this analysis, although we see hints that its mass lies somewhat above $160 \mathrm{GeV}$. We therefore only present here measurements of the masses of $\tilde{\ell}_{1}$ and $\tilde{\ell}_{2}$. The lack of direct observation of a third slepton is entirely to be expected for a spectrum with mixings such as ours, and for an analysis not attempting to reconstruct taus.

From the distribution given in figure 2, we measure $m_{\tilde{\ell}_{1}}=135.9 \pm 0.1 \mathrm{GeV}$. Given an integrated luminosity of $100 \mathrm{fb}^{-1}$, the high number of reconstructed $\tilde{\ell}_{1}$ allows us to measure the $\tilde{\ell}_{1}$ mass with small statistical error.

We expected to be able to measure the $\tilde{\ell}_{2}$ indirectly using indirect SS and indirect OS $\chi_{1}^{0}$ decays. In figure $4 \mathrm{a}$ we see the invariant mass distribution of OS $\tilde{\ell}_{1} e$ pairs. This distribution is peaked at $225.10 \pm 0.04 \mathrm{GeV}$. We expect this peak to be a combination of pure $\chi_{1}^{0}$ from direct decays and off-reconstructed $\chi_{1}^{0}$ from indirect decays. The OS $\tilde{\ell}_{1} \mu$ distribution of figure $4 \mathrm{~b}$ peaks at a mass roughly $5 \mathrm{GeV}$ lower. In figure 5 we see that both SS slepton-lepton invariant mass distributions have a mean close to the lower of the two OS peak positions. We therefore have a measurement consistent with ref. [33]: nearly 
degenerate $\tilde{\ell}_{1}$ and $\tilde{\ell}_{2}$ with the $\tilde{\ell}_{1}$ having a much greater selectron component than the $\tilde{\ell}_{2}$ (seen from the minimal contamination of the $\chi_{1}^{0}$ peak in the OS $\tilde{\ell}_{1} e$ distribution). With this hypothesis we can identify the position of the higher peak, namely the OS $\tilde{\ell}_{1} e$ peak, as the $\chi_{1}^{0}$. This gives us a $\chi_{1}^{0}$ mass of $225.10 \pm 0.04 \mathrm{GeV}$.

As explained in section 4.3.2, it is better to extract $E_{\text {shift }}$ from the SS distributions, as these only come from indirect decays. From figure $5 \mathrm{~b}$, we see that the shifted peak is at $219.39 \pm 0.06 \mathrm{GeV}$. We then find $E_{\text {shift }}=5.71 \pm 0.07 \mathrm{GeV}$. Using (4.1), we then find $\Delta m \equiv m_{\tilde{\ell}_{2}}-m_{\tilde{\ell}_{1}}=5.06 \pm 0.06 \mathrm{GeV}$, and so $m_{\tilde{\ell}_{2}}=141.0 \pm 0.1 \mathrm{GeV}$. Note that the uncertainty in $m_{\tilde{\ell}_{2}}$ is dominantly from the uncertainty in $m_{\tilde{\ell}_{1}}$.

Since the shifted neutralino peak appears in both the $\tilde{\ell}_{1}^{ \pm} \mu^{ \pm}$and $\tilde{\ell}_{1}^{ \pm} e^{ \pm}$invariant mass distributions, we have strong evidence that $\tilde{\ell}_{2}$ has both smuon and selectron components. Similarly, since we see peaks in both the $\tilde{\ell}_{1}^{ \pm} e^{\mp}$ and $\tilde{\ell}_{1}^{ \pm} \mu^{\mp}$ distributions, we conclude that $\tilde{\ell}_{1}$ has both selectron and smuon components. It is still unclear whether these states also have stau components. As we will see in section 6.2.1, we cannot rule out this possibility, but with some mild assumptions about the neutralino couplings to $\tilde{\ell}_{1,2}$ we can show that if there are non-zero stau components in $\tilde{\ell}_{1,2}$, they must be equal to each other.

The cleanest "flavor" measurement we can extract is based on the SS shifted neutralino peaks in figure 5 , since these are only sensitive to $\tilde{\ell}_{2}$. If we assume that the neutralino couplings to the first two generations are the same, then dividing the number of events $N_{\mathrm{SSe}}$ in the $\tilde{\ell}_{1} e$ peak of figure $5 \mathrm{a}$ by the number of events $N_{S S \mu}$ in the $\tilde{\ell}_{1} \mu$ peak of figure $5 \mathrm{~b}$, and adjusting for the different reconstruction efficiencies for electrons and muons, we find the ratio of selectron to smuon components of $\tilde{\ell}_{2}$ to be

$$
\left|U_{2 e} / U_{2 \mu}\right|^{2}=N_{\mathrm{SSe}} / N_{S S \mu}=0.071 \pm 0.010
$$

in good agreement with our input model.

One could try to rule out a third light slepton with a selectron or smuon component, based on the absence of a clear peak in our distributions. This would support the hypothesis that $\tilde{\ell}_{1,2}$ are selectron-smuon mixtures. Indeed, the fact that we only observe one shifted neutralino peak in the $\tilde{\ell}_{1} e$ and $\tilde{\ell}_{1} \mu$ distributions indicates that there is no such third slepton which is close in mass to $\tilde{\ell}_{1}$. Furthermore, although we have not shown these distributions here, if one does not impose the requirement that $\tilde{\ell}_{1}$ and one lepton reconstruct the neutralino peak, there is no clear peak in the $\tilde{\ell}_{1} \ell \ell^{\prime}$ invariant mass distributions, with $\ell, \ell^{\prime}=e, \mu$. However, the peak we see near $160 \mathrm{GeV}$ makes it hard to conclusively exclude such a third slepton, although, as we explained in section 4.4, this peak seems consistent with a pure stau state with mass above $160 \mathrm{GeV}$.

\subsection{The heavy sleptons $\tilde{\ell}_{4,5,6}$}

In principle, the invariant mass distributions of $\left(\tilde{\ell}_{1} \ell\right) \ell^{\prime}$ combinations contain a lot of information about $\tilde{\ell}_{4,5,6}$. There is however a lot of supersymmetric background which makes the identification of the peaks challenging. The cleanest peaks are obtained from OS combinations $\left(\tilde{\ell}_{1}^{ \pm} e^{\mp}\right) \ell^{\prime}$, where the $\tilde{\ell}_{1}^{ \pm} e^{\mp}$ pair reconstructs $m_{\chi_{1}^{0}}$, as shown in figure $6 \mathrm{ab}$. The OS combinations $\left(\tilde{\ell}_{1}^{ \pm} \mu^{\mp}\right) \ell^{\prime}$ of figure $6 \mathrm{~cd}$ also yield useful information, although the peaks 
are not as clean. Similarly, useful but not particularly clean peaks are found in the SS invariant mass distributions of figure 7 .

When we consider the invariant mass distributions of $\chi_{1}^{0} e^{ \pm}$combinations for $\chi_{1}^{0}$ reconstructed from $\tilde{\ell}_{1}^{\mp} e_{1}^{ \pm}$(figure $6 \mathrm{~b}$ ), we obtain a very clear peak which identifies $\tilde{\ell}_{4}$, with a mean value of $283.2 \pm 0.1 \mathrm{GeV}$. The peak near the $\tilde{\ell}_{6}$ mass produced by considering these combinations is questionable and contains much fewer events than the $\tilde{\ell}_{4}$ peak. The $\tilde{\ell}_{5}$ peak is negligible. This strongly suggests that $\tilde{\ell}_{4}$ has a dominant selectron component. The invariant mass distributions of $\chi_{1}^{0} e^{ \pm}$combinations for $\chi_{1}^{0}$ reconstructed from $\tilde{\ell}_{1}^{\mp} \mu_{1}^{ \pm}$(figure $6 \mathrm{~d}$ ) are not as convincing, but the presence again of a dominant peak near $\tilde{\ell}_{4}$ further supports $\tilde{\ell}_{4}$ having a strong selectron component.

When we consider the invariant mass distributions of $\chi_{1}^{0} \mu^{ \pm}$combinations (figure 6a), one peak is present, which identifies $\tilde{\ell}_{6}$. We see an additional excess of events at a lower mass, which could be evidence for $\tilde{\ell}_{5}$. In figure $7 \mathrm{c}$ a similar excess, shifted to lower mass, supports the hypothesis that this excess of events is more than just a fluctuation of the background and is indeed $\tilde{\ell}_{5}$. We have already described a lack of evidence for selectron components in these two mass states. The mass of the clear heavier peak, $\tilde{\ell}_{6}$, is $343.3 \pm$ $0.5 \mathrm{GeV}$. If we assume the excess at lower mass is from $\tilde{\ell}_{5}$, then the peak describes a mass of $307 \pm 5 \mathrm{GeV}$. These two masses are a reasonable measure of the true slepton masses given that the model for the background is in this instance not ideal. We would, however, need a greater number of events to truly believe that the excess near $305 \mathrm{GeV}$ is the $\tilde{\ell}_{5}$ and not fluctation of the background.

Assuming that $\chi_{2}^{0}$ is predominantly gaugino, and that $\tilde{\ell}_{5}$ and $\tilde{\ell}_{6}$ have identical quantum numbers, the $\chi_{2}^{0}$ branching ratios to these two sleptons can only differ due to phase space effects. We know that $\tilde{\ell}_{5,6}$ have negligible selectron components, so can only be smuon-stau mixtures. The ratio of the number of events in the $\tilde{\ell}_{5}$ peak to the number of events in the $\tilde{\ell}_{6}$ peak of figure 6 a therefore gives the smuon-stau mixing in these states up to the phasespace factor. Although the compounded reconstruction errors and ignorance of systematics make exact results from this analysis suspect, we can conclude that this mixing is $\mathcal{O}(1)$, if we support the excess of events near $307 \mathrm{GeV}$ being a $\tilde{\ell}_{5}$ mass peak.

\section{Discussion}

We now reflect on the measurements we have made and on the measurements we have been unable to make. In what follows, we consider how the lessons learned from considering this particular supersymmetry model generalize to a wider range of supersymmetric models. We also consider in this section what we might be able to deduce if, in addition to these experimental observations, we make the assumption that we are expecting to observe the six slepton states of an MSSM-type model.

\subsection{Mass measurements}

Out of the six sleptons, we have been able to identify in a rather convincing way the masses of four: $\tilde{\ell}_{1}, \tilde{\ell}_{2}, \tilde{\ell}_{4}$ and $\tilde{\ell}_{6}$. We have evidence for $\tilde{\ell}_{5}$, though given the statistics available it is not clear that were this peak truly observed in an experiment it would necessarily 
be more than background fluctuations. The $\tilde{\ell}_{2}$ mass has been determined indirectly using the neutralino shifted peak, whereas the other sleptons are found by direct measurement. In all five cases the masses we obtain are in reasonable or good agreement with the true slepton masses. We see hints for $\tilde{\ell}_{3}$ but cannot measure its mass conclusively because of its dominant stau component, and because it happens to be close in mass to $\tilde{\ell}_{2}$.

The correct determination of as many as five of the six sleptons tells us that for fully reconstructible supersymmetric decay chains ending in stable charged NLSPs, working up the decay chain and reconstructing invariant mass distributions in stages is a promising way of measuring superpartner masses in the slepton sector. We have seen that, even with nearly degenerate states, indirect methods do exist which may still render these slepton masses measurable.

\subsection{Mixings}

\subsubsection{Light sleptons}

Our best mixing measurement is of the $e-\mu$ ratio in $\tilde{\ell}_{2}$, shown in (5.1). If we assume that $\tilde{\ell}_{1,2}$ are selectron-smuon mixtures, with no stau components, we have

$$
\left(\begin{array}{c}
\tilde{\ell}_{1} \\
\tilde{\ell}_{2}
\end{array}\right)=\left(\begin{array}{cc}
\cos \theta_{12}^{R} & \sin \theta_{12}^{R} \\
-\sin \theta_{12}^{R} & \cos \theta_{12}^{R}
\end{array}\right)\left(\begin{array}{c}
\tilde{e}_{R} \\
\tilde{\mu}_{R}
\end{array}\right) .
$$

With this assumption, our measurement of (5.1) implies

$$
\sin ^{2} \theta_{12}^{R}=0.066 \pm 0.009
$$

How well can we test the assumption that $\tilde{\ell}_{1,2}$ have no stau component? Clearly a direct determination cannot be done in an analysis that does not look at $\tau$ leptons, but it is interesting to ask whether we can use the information we have on $\tilde{\ell}_{1}$ and $\tilde{\ell}_{2}$ to argue this. As we will now see, with some mild assumptions about the model, we can show that $\tilde{\ell}_{1}$ and $\tilde{\ell}_{2}$ can only have equal stau components, i.e., $\left|U_{1 \tau}\right|=\left|U_{2 \tau}\right|$. We cannot exclude, however, $\left|U_{1 \tau}\right|=\left|U_{2 \tau}\right| \neq 0$.

We begin by assuming that the neutralino coupling to the three light sleptons is flavorblind, an assumption that is valid not only in our model, but generically in the types of models we are considering. In the mass distributions of figures 4 and 5 , we take the number of events in the OS electron (muon) peak to be $N_{\text {OSe }}\left(N_{O S \mu}\right)$. Then

$$
\begin{aligned}
N_{\mathrm{SSe}} & =N f_{\mathrm{SS}}\left|U_{2 e}\right|^{2} \\
N_{S S \mu} & =N f_{\mathrm{SS}}\left|U_{2 \mu}\right|^{2} \\
N_{\mathrm{OSe}} & =N\left[f_{12}\left|U_{1 e}\right|^{2}+\left(1-f_{\mathrm{SS}}\right)\left|U_{2 e}\right|^{2}\right] \\
N_{O S \mu} & =N\left[f_{12}\left|U_{1 \mu}\right|^{2}+\left(1-f_{\mathrm{SS}}\right)\left|U_{2 \mu}\right|^{2}\right],
\end{aligned}
$$

where $N$ is the total number of neutralino decays to $\tilde{\ell}_{2}$, and $f_{\mathrm{SS}}$ is the fraction of $\tilde{\ell}_{2} \rightarrow \tilde{\ell}_{1}$ decays in which the slepton charge flips $\operatorname{sign}\left(\tilde{\ell}_{2}^{ \pm} \rightarrow \tilde{\ell}_{1}^{\mp}\right)$. We include the factor $f_{12} \approx 1$ 
to account for the different masses of $\tilde{\ell}_{1}$ and $\tilde{\ell}_{2}$. Assuming that we already know that the neutralino is a fermion and the sleptons are scalars, we have

$$
f_{12}=\left(\frac{1-m_{1}^{2} / M^{2}}{1-m_{2}^{2} / M^{2}}\right)^{2},
$$

where one power comes from phase space, and the other one from the matrix element for the $\chi_{1}^{0} \rightarrow \tilde{\ell} \ell$ decay.

It is useful to combine these four equations to obtain two combinations that are independent of $N$ and $f_{\mathrm{SS}}$. One such combination is the ratio $N_{\mathrm{SSe}} / N_{S S \mu}$, which we already used to extract $\left|U_{2 e}\right| /\left|U_{2 \mu}\right|$. The other one is

$$
\frac{N_{e}}{N_{\mu}}=\frac{N_{\mathrm{SSe}}+N_{\mathrm{OSe}}}{N_{S S \mu}+N_{O S \mu}}=\frac{f_{12}\left|U_{1 e}\right|^{2}+\left|U_{2 e}\right|^{2}}{f_{12}\left|U_{1 \mu}\right|^{2}+\left|U_{2 \mu}\right|^{2}} .
$$

For the 2-state assumption, using the value of $\sin ^{2} \theta_{12}^{R}$ we found above, the theoretical prediction is $N_{e} / N_{\mu} \approx f_{12}=1.09$, in reasonable agreement with the data,

$$
N_{e} / N_{\mu}=1.16 \pm 0.02 .
$$

It is easy to see, however, that this agreement would persist for any

$$
\left|U_{2 e}\right|,\left|U_{1 \mu}\right| \ll\left|U_{1 e}\right| \simeq\left|U_{2 \mu}\right|,
$$

which, for $\left|U_{1 \tau}\right|,\left|U_{2 \tau}\right| \nless\left|U_{1 e}\right|,\left|U_{2 \mu}\right|$, implies (by unitarity) that $\left|U_{1 \tau}\right| \simeq\left|U_{2 \tau}\right|$.

\subsubsection{Heavy sleptons}

It is encouraging that, despite the $\tilde{\ell}_{4}$ being produced high up in our decay chains, we have good evidence that $\tilde{\ell}_{4}$ has a very strong selectron component and negligible smuon component from the strong peaks in the $\chi_{1}^{0} e$ invariant mass distributions and the absence of a peak in the $\chi_{1}^{0} \mu$ invariant mass distributions. For $\tilde{\ell}_{5}$ and $\tilde{\ell}_{6}$ we know that these have strong smuon components but negligible selectron components. Assuming that these states are left-handed sleptons, and that the $\chi_{2}^{0}$ is a gaugino, we can deduce that the smuon-stau mixing in these states is of $\mathcal{O}(1)$. Assuming further that $\tilde{\ell}_{4}$ is a left-handed slepton, we can also conclude that it is predominantly a selectron.

\section{Conclusions}

The understanding of slepton masses and mixings that we have arrived at, based on experimental evidence, is summarized in table 1 . The results are very close to the assumed underlying input model. We have taken a "data-driven" approach, in which we try to infer from the data the masses and flavor compositions of new particles, rather than fitting the parameters of a particular model to the data. The methods we described are specific to models with metastable charged particles and, therefore, no missing energy.

The most obvious way to identify the sleptons in our model (which we initially tried) would be to look for peaks in the NLSP plus two leptons invariant-mass distributions. This 
does not work very well, however, either because some of the relevant leptons are too soft or because of the large supersymmetric combinatoric backgrounds. Instead, we showed that the best mass measurements in these scenarios can be obtained by reconstructing the spectrum in stages, starting from the bottom up. For example, rather than discovering a heavy slepton by searching for a peak in the invariant mass distribution of the NLSP plus two leptons, we first identify the lightest neutralino as a peak in the NLSP plus lepton distribution, and then combine this neutralino with one additional lepton to obtain the heavier slepton peak. We also demonstrated that nearly-degenerate particles can be resolved indirectly, using the shifted peak of their mother particle.

We then outlined methods for measuring the slepton flavor compositions, essentially by counting experiments. We were able to qualitatively infer the flavor makeup of the sleptons, and to quantitatively measure one mixing.

The charged NLSP is a crucial ingredient in our analysis, yet the model we chose also has some unfavorable features:

1. The two lightest sleptons are almost degenerate.

2. The $e-\mu$ mixing in these sleptons is small.

3. One slepton, $\tilde{\ell}_{3}$, is an almost pure stau state, and is quite close in mass to $\tilde{\ell}_{1,2}$, so that the peak it gives in the NLSP plus $e / \mu$ distributions is very close to the $\tilde{\ell}_{2}$ peak.

We have also not used any information from tau leptons. Given these difficult features and the pessimistic assumption about taus, the amount of information we were able to extract is encouraging.

The analysis we presented is certainly preliminary. If our toy model were real data, one would want to perform detailed measurements to confirm that the observed events were supersymmetric, establish the gaugino identities of the neutralinos, and explore the squark and gluino sector. One could then fit the flavor parameters of the model to the data, and flesh out the details of the picture we outlined here.

We conclude that, under favorable but not outrageously optimistic circumstances (unless we think of discovering supersymmetry as outrageously optimistic), it is possible that measurements made at the Large Hadron Collider will allow us to determine a great deal about the slepton spectrum and flavor decomposition, and constrain, and possibly clarify, the flavor structures of both supersymmetry and the standard model.

\section{Acknowledgments}

We thank James Frost and Are Raklev for technical assistance and Kfir Blum for discussions. YS thanks the Aspen Center for Physics, where part of this work was completed. The research of JLF, YN, YS and IG was supported in part by the United States-Israel Binational Science Foundation (BSF) under Grant No. 2006071. The work of JLF, DS, and FY was supported in part by NSF Grant No. PHY-0653656. STF and CGL acknowledge the support of the United Kingdom's Science and Technology Facilities Council, Peterhouse and the University of Cambridge. The work of IG and YS was supported in part 
by the Israel Science Foundation (ISF) under Grant No. 1155/07. The work of YN is supported by the Israel Science Foundation (ISF) under Grant No. 377/07, the German-Israeli Foundation for scientific research and development (GIF), and the Minerva Foundation.

\section{A Model details}

\section{A.1 Input parameters}

As discussed in section 2, the model used for this analysis is a hybrid model in which slepton masses receive contributions from both gauge- and gravity-mediated supersymmetry breaking. Gauge-mediation provides the primary, flavor-universal contributions to soft masses, and gravity-mediation supplements these with flavor-violating contributions. The slepton soft mass matrices then have the form

$$
\begin{aligned}
M_{\tilde{\nu}}^{2} & =m_{\tilde{\ell}}^{2} \mathbf{1}+x m_{\tilde{\ell}}^{2} X_{L} \\
M_{\tilde{\ell}_{L}}^{2} & =m_{\tilde{\ell}}^{2} \mathbf{1}+m_{E} m_{E}^{\dagger}+x m_{\tilde{\ell}}^{2} X_{L} \\
M_{\tilde{\ell}_{R}}^{2} & =m_{\tilde{R}}^{2} \mathbf{1}+m_{E}^{\dagger} m_{E}+x m_{\tilde{\ell}}^{2} X_{R},
\end{aligned}
$$

where $m_{\tilde{\ell}}^{2}$ and $m_{\tilde{R}}^{2}$ are the gauge-mediated, flavor-conserving contributions to the left- and right-handed sleptons, $m_{E}$ is the lepton mass matrix, and $x$ is the ratio between gravityand gauge-mediated contributions. The symmetric matrices $X_{L}$ and $X_{R}$ parameterize the gravity-mediated flavor-violating effects for left- and right-handed sleptons, with the flavor structure determined by the Froggatt-Nielsen mechanism [14].

For the model analyzed here, the flavor-conserving gauge-mediated contributions are specified by the standard GMSB parameters

$$
N_{5}=5, M_{\mathrm{m}}=4.6 \cdot 10^{6} \mathrm{GeV}, \Lambda=3.4 \cdot 10^{4} \mathrm{GeV}, C_{\text {grav }}=1, \tan \beta=10, \mu>0 .
$$

The gravity-mediated, flavor-violating contributions are governed by a $\mathrm{U}(1) \times \mathrm{U}(1)$ horizontal symmetry under which the lepton superfields have the charges

$$
L_{1}(2,0), L_{2}(0,2), L_{3}(0,2) ; \quad \bar{E}_{1}(2,1), \bar{E}_{2}(2,-1), \bar{E}_{3}(0,-1) .
$$

The resulting flavor-violating masses are generated using appropriate powers of the Froggatt-Nielsen expansion parameter $\lambda$ with $\mathcal{O}(1)$ coefficients for each term in the matrix. The resulting flavor-violating contributions for the sleptons are

$$
\begin{aligned}
X_{L} & =\left(\begin{array}{rrr}
-3.4989 \lambda^{0} & -1.2001 \lambda^{4} & 0.4059 \lambda^{4} \\
-1.2001 \lambda^{4} & -1.2705 \lambda^{0} & 1.1746 \lambda^{0} \\
0.4059 \lambda^{4} & 1.1746 \lambda^{0} & 1.4293 \lambda^{0}
\end{array}\right) \\
X_{R} & =\left(\begin{array}{rrr}
-1.0368 \lambda^{0} & 0.9976 \lambda^{2} & -0.06188 \lambda^{4} \\
0.9976 \lambda^{2} & -0.8616 \lambda^{0} & 0.2204 \lambda^{2} \\
-0.06188 \lambda^{4} & 0.2204 \lambda^{2} & 0.6544 \lambda^{0}
\end{array}\right),
\end{aligned}
$$

and the lepton mass matrix is given by 


\begin{tabular}{|c|c|r|r|r|r|r|c|}
\hline & Mass $(\mathrm{GeV})$ & \multicolumn{1}{|c|}{$\tilde{e}_{R}$} & $\tilde{\mu}_{R}$ & $\tilde{\tau}_{R}$ & $\tilde{e}_{L}$ & $\tilde{\mu}_{L}$ & $\tilde{\tau}_{L}$ \\
\hline$\tilde{\ell}_{6}$ & 343.53 & 0 & -0.0043 & -0.0445 & -0.0005 & 0.8789 & 0.4749 \\
$\tilde{\ell}_{5}$ & 303.41 & 0 & 0.0028 & -0.1154 & -0.0009 & -0.4767 & 0.8715 \\
$\tilde{\ell}_{4}$ & 282.86 & 0 & 0 & -0.0002 & 1.0000 & 0 & 0.0010 \\
$\tilde{\ell}_{3}$ & 168.30 & -0.0012 & 0.0238 & 0.9920 & 0 & -0.0159 & 0.1226 \\
$\tilde{\ell}_{2}$ & 140.78 & -0.2551 & 0.9666 & -0.0231 & 0 & 0.0053 & -0.0032 \\
$\tilde{\ell}_{1}$ & 135.83 & 0.9669 & 0.2551 & -0.0048 & 0 & 0.0014 & -0.0007 \\
\hline
\end{tabular}

Table 2. Physical Slepton Masses and Flavor Composition. Elements of the mixing matrix with values less than $10^{-4}$ have been set to zero.

\begin{tabular}{|c|c|r|r|c|}
\hline & Mass $(\mathrm{GeV})$ & \multicolumn{1}{|c|}{$\tilde{\nu}_{e}$} & \multicolumn{1}{c|}{$\tilde{\nu}_{\mu}$} & $\tilde{\nu}_{\tau}$ \\
\hline$\tilde{\nu}_{3}$ & 334.44 & -0.0005 & 0.8695 & 0.4939 \\
$\tilde{\nu}_{2}$ & 293.82 & -0.0009 & -0.4939 & 0.8695 \\
$\tilde{\nu}_{1}$ & 271.37 & 1.0000 & 0 & 0.0010 \\
\hline
\end{tabular}

Table 3. Physical Sneutrino Masses and Flavor Composition. Elements of the mixing matrix with values less than $10^{-4}$ have been set to zero.

$$
m_{E}=\left\langle\phi_{d}\right\rangle \lambda\left(\begin{array}{rrr}
-0.3838 \lambda^{4} & 0 & 0 \\
0.8706 \lambda^{4} & -1.8682 \lambda^{2} & -1.5408 \lambda^{0} \\
1.0450 \lambda^{4} & 0.3574 \lambda^{2} & 1.8554 \lambda^{0}
\end{array}\right) .
$$

We ignore the neutrino mass matrix for our purposes, as neutrino flavor is unobservable in colliders and thus unimportant for this study. We take $x=0.1$ and $\lambda=0.2$.

\section{A.2 Spectrum}

Given these input parameters, the full supersymmetric spectrum and flavor-general decay branching ratios are generated by the program SpICE [17]. The SPICE input file used is

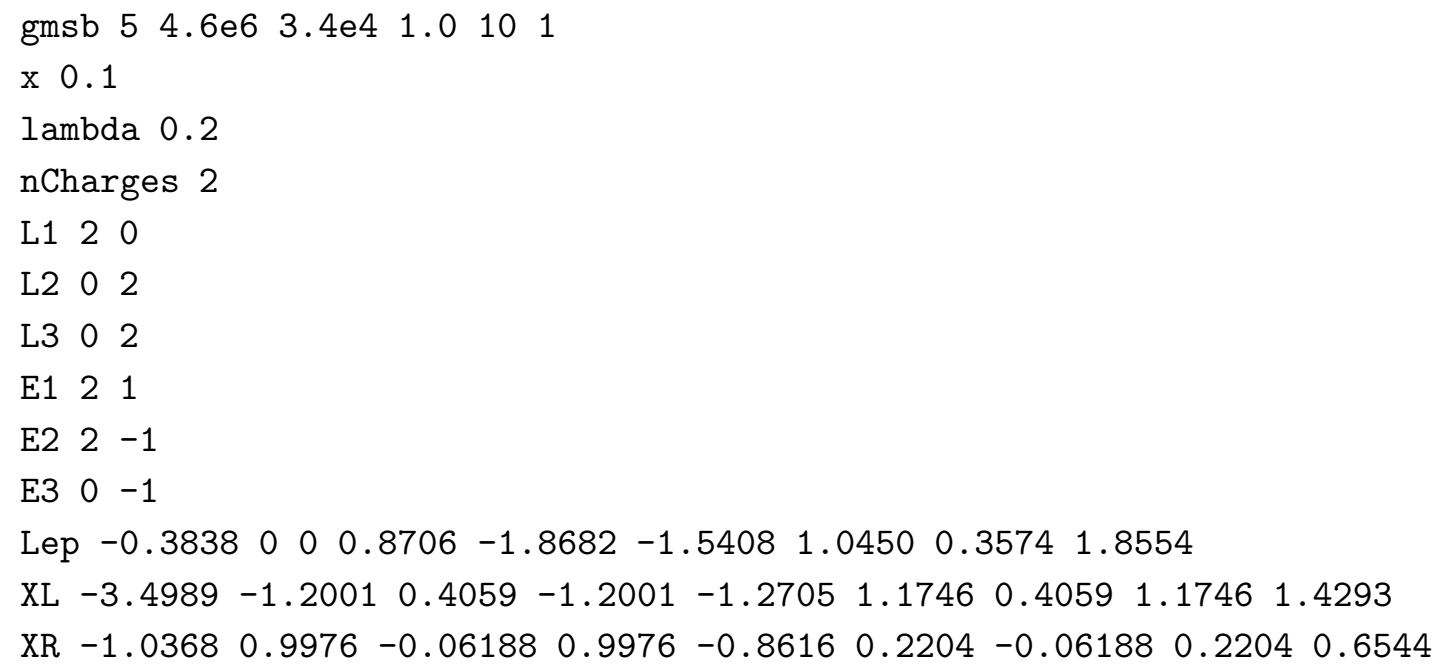




\begin{tabular}{|c|r|c|r|}
\hline & Mass $(\mathrm{GeV})$ & & Mass $(\mathrm{GeV})$ \\
\hline$\tilde{d}_{L}$ & 1093.69 & $h^{0}$ & 111.50 \\
$\tilde{u}_{L}$ & 1086.02 & $H^{0}$ & 562.02 \\
$\tilde{s}_{L}$ & 1093.58 & $A^{0}$ & 561.68 \\
$\tilde{c}_{L}$ & 1086.02 & $H^{+}$ & 567.61 \\
$\tilde{b}_{1}$ & 1039.56 & $\chi_{1}^{0}$ & 224.83 \\
$\tilde{t}_{1}$ & 944.91 & $\chi_{2}^{0}$ & 398.87 \\
$\tilde{d}_{R}$ & 1050.83 & $\chi_{3}^{0}$ & 470.73 \\
$\tilde{u}_{R}$ & 1053.09 & $\chi_{4}^{0}$ & 521.28 \\
$\tilde{s}_{R}$ & 1050.83 & $\chi_{1}^{+}$ & 399.53 \\
$\tilde{c}_{R}$ & 1053.09 & $\chi_{2}^{+}$ & 522.02 \\
$\tilde{b}_{2}$ & 1050.92 & $\tilde{g}$ & 1225.74 \\
$\tilde{t}_{2}$ & 1061.48 & $\tilde{G}$ & $\sim 40$ \\
\hline
\end{tabular}

Table 4. Particle Spectrum. Physical masses of the squarks, gluino, Higgs bosons, charginos, neutralinos, and gravitino.

The masses of the six slepton mass eigenstates, as well as the rotation matrix relating the slepton mass and gauge eigenstates, are presented in table 2. These are discussed in section 2, with a graphical representation given in figure 1 . The sneutrino masses and flavor compositions are given in table 3 .

The masses of the remaining MSSM particles are given in table 4. The SM-like Higgs boson is below the current bounds, but it and its precise mass play no role in the signals studied here. The gravitino mass is not precisely specified. The dominant contribution to its mass is from gravity-mediation, and so we expect $m_{\tilde{G}} \sim \sqrt{x} m_{\tilde{\ell}}$. This guarantees that decays to the gravitino take place outside collider detectors and the signals we study are insensitive to the precise value of $m_{\tilde{G}}$. The general mass ordering of the lightest states is therefore

$$
m_{\tilde{G}} \ll m_{\tilde{\ell}_{1,2,3}}<m_{\chi_{1}^{0}}<m_{\tilde{\ell}_{4,5,6}}<m_{\chi_{2}^{0}} .
$$

The decays from the lightest neutralino to the light sleptons, and from the light sleptons to other light sleptons, are also shown in figure 1.

Open Access. This article is distributed under the terms of the Creative Commons Attribution Noncommercial License which permits any noncommercial use, distribution, and reproduction in any medium, provided the original author(s) and source are credited.

\section{References}

[1] N. Arkani-Hamed, H.-C. Cheng, J.L. Feng and L.J. Hall, Probing lepton flavor violation at future colliders, Phys. Rev. Lett. 77 (1996) 1937 [hep-ph/9603431] [SPIRES].

[2] N. Arkani-Hamed, J.L. Feng, L.J. Hall and H.-C. Cheng, CP violation from slepton oscillations at the LHC and NLC, Nucl. Phys. B 505 (1997) 3 [hep-ph/9704205] [SPIRES]. 
[3] K. Agashe and M. Graesser, Signals of supersymmetric lepton flavor violation at the LHC, Phys. Rev. D 61 (2000) 075008 [hep-ph/9904422] [SPIRES].

[4] J. Hisano, R. Kitano and M.M. Nojiri, Slepton oscillation at Large Hadron Collider, Phys. Rev. D 65 (2002) 116002 [hep-ph/0202129] [SPIRES].

[5] T. Goto, K. Kawagoe and M.M. Nojiri, Study of the slepton non-universality at the CERN Large Hadron Collider, Phys. Rev. D 70 (2004) 075016 [Erratum ibid. D 71 (2005) 059902] [hep-ph/0406317] [SPIRES].

[6] R. Kitano, A clean slepton mixing signal at the LHC, JHEP 03 (2008) 023 [arXiv: 0801.3486] [SPIRES].

[7] B.C. Allanach, J.P. Conlon and C.G. Lester, Measuring Smuon-Selectron mass splitting at the CERN LHC and patterns of supersymmetry breaking, Phys. Rev. D 77 (2008) 076006 [arXiv:0801.3666] [SPIRES].

[8] S. Kaneko, J. Sato, T. Shimomura, O. Vives and M. Yamanaka, Measuring lepton flavour violation at LHC with long-lived slepton in the coannihilation region, Phys. Rev. D 78 (2008) 116013 [arXiv:0811.0703] [SPIRES].

[9] A. De Simone, J. Fan, V. Sanz and W. Skiba, Leptogenic supersymmetry, Phys. Rev. D 80 (2009) 035010 [arXiv: 0903.5305] [SPIRES].

[10] J.L. Feng, C.G. Lester, Y. Nir and Y. Shadmi, The standard model and supersymmetric flavor puzzles at the Large Hadron Collider, Phys. Rev. D 77 (2008) 076002 [arXiv: 0712.0674] [SPIRES].

[11] Z. Chacko and E. Ponton, Yukawa deflected gauge mediation, Phys. Rev. D 66 (2002) 095004 [hep-ph/0112190] [SPIRES].

[12] R.N. Mohapatra, N. Okada and H.-B. Yu, $\nu$-GMSB with type III seesaw and phenomenology, Phys. Rev. D 78 (2008) 075011 [arXiv:0807.4524] [SPIRES].

[13] J.L. Feng and T. Moroi, Tevatron signatures of longlived charged sleptons in gauge mediated supersymmetry breaking models, Phys. Rev. D 58 (1998) 035001 [hep-ph/9712499] [SPIRES].

[14] C.D. Froggatt and H.B. Nielsen, Hierarchy of quark masses, cabibbo angles and cp-violation, Nucl. Phys. B 147 (1979) 277 [SPIRES].

[15] Y. Nir and N. Seiberg, Should squarks be degenerate?, Phys. Lett. B 309 (1993) 337 [hep-ph/9304307] [SPIRES].

[16] G.G. Ross and O. Vives, Yukawa structure, flavour and CP-violation in supergravity, Phys. Rev. D 67 (2003) 095013 [hep-ph/0211279] [SPIRES].

[17] G. Engelhard, J.L. Feng, I. Galon, D. Sanford and F. Yu, SPICE: simulation package for including flavor in collider events, Comput. Phys. Commun. 181 (2010) 213 [arXiv: 0904.1415] [SPIRES].

[18] B.C. Allanach, SOFTSUSY: A C++ program for calculating supersymmetric spectra, Comput. Phys. Commun. 143 (2002) 305 [hep-ph/0104145] [SPIRES].

[19] B.C. Allanach and M.A. Bernhardt, Including R-parity violation in the numerical computation of the spectrum of the minimal supersymmetric standard model: SOFTSUSY3.0, Comput. Phys. Commun. 181 (2010) 232 [arXiv:0903.1805] [SPIRES]. 
[20] A. Djouadi, M.M. Muhlleitner and M. Spira, Decays of supersymmetric particles: the program SUSY-HIT (SUspect-SdecaY-HDECAY-InTerface), Acta Phys. Polon. B 38 (2007) 635 [hep-ph/0609292] [SPIRES].

[21] J.L. Feng, I. Galon, D. Sanford, Y. Shadmi and F. Yu, Three-body decays of sleptons with general flavor violation and left-right mixing, Phys. Rev. D 79 (2009) 116009 [arXiv:0904.1416] [SPIRES].

[22] G. Marchesini et al., HERWIG: a monte carlo event generator for simulating hadron emission reactions with interfering gluons. Version 5.1 - April 1991, Comput. Phys. Commun. 67 (1992) 465 [SPIRES].

[23] G. Corcella et al., HERWIG 6.5: an event generator for Hadron Emission Reactions with interfering gluons (including supersymmetric processes), JHEP 01 (2001) 010 [hep-ph/0011363] [SPIRES].

[24] P.Z. Skands et al., SUSY les houches accord: interfacing SUSY spectrum calculators, decay packages and event generators, JHEP 07 (2004) 036 [hep-ph/0311123] [SPIRES].

[25] E. Richter-Was, AcerDET: A particle level fast simulation and reconstruction package for phenomenological studies on high $p_{T}$ physics at LHC, hep-ph/0207355 [SPIRES].

[26] ATLAS collaboration, G. Aad et al., The ATLAS Experiment at the CERN Large Hadron Collider, 2008 JINST 3 S08003 [SPIRES].

[27] J.R. Ellis, A.R. Raklev and O.K. Oye, Gravitino dark matter scenarios with massive metastable charged sparticles at the LHC, JHEP 10 (2006) 061 [hep-ph/0607261] [SPIRES].

[28] S. Tarem, S. Bressler, H. Nomoto and A. Di Mattia, Trigger and reconstruction for heavy long-lived charged particles with the ATLAS detector, Eur. Phys. J. C 62 (2009) 281 [SPIRES].

[29] J.L. Feng, A. Rajaraman and B.T. Smith, Minimal supergravity with $m_{0}^{2}<0$, Phys. Rev. D 74 (2006) 015013 [hep-ph/0512172] [SPIRES].

[30] A. Rajaraman and B.T. Smith, Discovering SUSY with $m(0)^{2}<0$ in the first CERN LHC physics run, Phys. Rev. D 75 (2007) 115015 [hep-ph/0612235] [SPIRES].

[31] A. Rajaraman and B.T. Smith, Determining spins of metastable sleptons at the Large Hadron Collider, Phys. Rev. D 76 (2007) 115004 [arXiv:0708.3100] [SPIRES].

[32] The CMS collaboration, Search for heavy stable charged particles with 100/pb and 1/fb in the CMS experiment, CMS-PAS-EXO-08-003.

[33] J.L. Feng, S.T. French, C.G. Lester, Y. Nir and Y. Shadmi, The shifted peak: resolving nearly degenerate particles at the LHC, Phys. Rev. D 80 (2009) 114004 [arXiv:0906.4215] [SPIRES]. 